\title{
Solving Fully Fuzzy Nonlinear Eigenvalue Problems of Damped Spring-Mass Structural Systems Using Novel Fuzzy-Affine Approach
}

\author{
S. Rout $^{1}$ and S. Chakraverty ${ }^{1, *}$
}

\begin{abstract}
The dynamic analysis of damped structural system by using finite element method leads to nonlinear eigenvalue problem (NEP) (particularly, quadratic eigenvalue problem). In general, the parameters of NEP are considered as exact values. But in actual practice because of different errors and incomplete information, the parameters may have uncertain or vague values and such uncertain values may be considered in terms of fuzzy numbers. This article proposes an efficient fuzzy-affine approach to solve fully fuzzy nonlinear eigenvalue problems (FNEPs) where involved parameters are fuzzy numbers viz. triangular and trapezoidal. Based on the parametric form, fuzzy numbers have been transformed into family of standard intervals. Further due to the presence of interval overestimation problem in standard interval arithmetic, affine arithmetic based approach has been implemented. In the proposed method, the FNEP has been linearized into a generalized eigenvalue problem and further solved by using the fuzzy-affine approach. Several application problems of structures and also general NEPs with fuzzy parameters are investigated based on the proposed procedure. Lastly, fuzzy eigenvalue bounds are illustrated with fuzzy plots with respect to its membership function. Few comparisons are also demonstrated to show the reliability and efficacy of the present approach.
\end{abstract}

Keywords: Fuzzy nonlinear eigenvalue problem, fuzzy set theory, affine arithmetic, interval overestimation problem, triangular fuzzy number, trapezoidal fuzzy number, fuzzy-affine approach.

\section{Introduction}

The nonlinear eigenvalue problem (NEP) is an emerging class of dynamical problems arising from a variety of science and engineering applications. Let us consider the function $N: \Omega \rightarrow C^{m \times m}$, where $\Omega \subseteq C$ is an open set. The NEP is to find the scalars and nonzero vectors $(x, \lambda) \in C^{m} \times \Omega$ such that

$N(\lambda) x=0$.

where $\lambda$ is called the eigenvalue and $x$ is known as the corresponding eigenvector.

\footnotetext{
${ }^{1}$ Department of Mathematics, National Institute of Technology Rourkela, Odisha, India.

${ }^{*}$ Corresponding Author: S. Chakraverty. Email: sne_chak@yahoo.com.
} 
There are wide varieties of applications of NEPs in the dynamic analysis of various science and engineering problems viz. structural mechanics and acoustic systems, simulation of electrical circuits, modeling micro-electronic mechanical systems, fluid mechanics and signal processing, etc. NEP plays an important role in the application of structural dynamics. In general, dynamic analysis of structural problems of damped spring-mass system gets converted into NEP (particularly quadratic eigenvalue problem (QEP)).

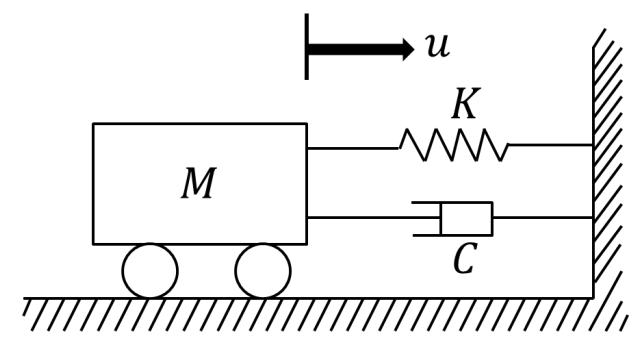

Figure 1: One degree-of-freedom damped spring-mass structural system

The equation of motion for the structural ambient vibrational problems of the system given in Fig. 1 may be written as

$M \ddot{u}(t)+C \dot{u}(t)+K u(t)=0$,

where $M$ is mass matrix, $C$ the damping matrix, $K$ stiffness matrix. Substituting $u=x e^{i \omega \cdot t}$ in the governing equation of motion (Eq. (2)) due to dynamic analysis of structures may be reduced to a QEP

$\left(K+\lambda C+\lambda^{2} M\right) x=0$,

where $\lambda=i \omega$ stands for the eigenvalue and $x$ is the corresponding eigenvector of the above problem.

Many investigations are carried out by various researchers to handle the crisp NEPs. Basic theories and properties of matrix structural analysis are presented by Przemieniecki [Przemieniecki (1968)]. Further, Gohberg et al. [Gohberg, Lancaster and Rodman (1982)] discussed various concepts and propertied of matrix polynomials. Rajakumar [Rajakumar (1993)] developed the Lanczos algorithm for the solution of QEPs in engineering applications. The backward error analysis of polynomial eigenvalue problems (which is a particular type of NEP) is explained by Tisseur [Tisseur (2000)]. Tisseur et al. [Tisseur and Meerbergen (2001)] illustrated a review work on solving crisp QEPs given by Eq. (3) using several numerical techniques. Their work is based upon the linearization of the NEP into standard eigenvalue problem (SEP) and generalized eigenvalue problem (GEP). Mehrmann et al. [Mehrmann and Watkins (2002)] studied the linearization procedure to solve polynomial eigenvalue problems with Hamiltonian structure. Structured eigenvalue method for the computation of corner singularities in 3D anisotropic elastic structures has been studied by Apel et al. [Apel, Mehrmann and Watkins (2002)]. Further, Bai et al. [Bai and Su (2005)] proposed a second-order Arnoldi method (SOAR) to solve the crisp QEP. Study of a special form of NEP known as rational eigenvalue problems and its solution via linearization technique was presented by $\mathrm{Su}$ et al. [Su and Bai (2011)]. Wetherhold et al. [Wetherhold and Padliya (2014)] designed different aspects of 
nonlinear vibration analysis of rectangular orthotropic membranes. Again a backward error analysis of polynomial eigenvalue problems solved by linearization has been studied by Lawrence et al. [Lawrence, Van Barel and Van Dooren (2016)]. Solov'ev et al. [Solov'ev and Solov'ev (2018)] developed a finite element approximation of the minimal eigenvalue of a NEP. NEP is solved by Saad et al. [Saad, El-Guide and Miedlar (2019)] using a rational approximation method. The linearised and nonlinearised solutions of nonlinear multiparameter eigenvalue problems are discussed by Kurseeva et al. [Kurseeva, Tikhov, and Valovik (2019)]. Bender et al. [Bender, Komijani and Wang (2019)] studied NEP for generalized Painlevé equations. Moreover, Newton method is widely used to solve NEP having crisp parameters. For instance, Kressner [Kressner (2009)] developed a block Newton method for the solution of NEPs. An improved Newton method was also proposed by Fazeli et al. [Fazeli and Rabiei (2016)] to solve NEPs. Further, Gao et al. [Gao, Yang and Meza (2009)] described the solution of a class of NEPs by Newton's method.

In general, all the parameters associated with the NEP are considered as crisp or exact values. But in real practice, because of different errors and incomplete information, one may have only the vague and imprecise parameters which are uncertain in nature. Fuzzy set theory and interval analysis are two major fields to handle these parameters. In fuzzy set theory, the fuzzy numbers may be converted into corresponding interval forms using $\eta$-cut method. Few literature studies related to the basic concepts and properties of fuzzy set theory are discussed here. Zadeh [Zadeh (1965)] first introduced the concepts of fuzzy sets and fuzzy numbers. Many excellent books have been written by few authors viz., Dubois [Dubois (1980)], Kaufmann et al. [Kaufmann and Gupta (1988)], Zimmermann [Zimmermann (2001)], Hanss [Hanss (2005)], Zadeh et al. [Zadeh, Fu and Tanaka (2014)], Chakraverty et al. [Chakraverty, Tapaswini and Behera (2016)] and Chakraverty et al. [Chakraverty and Perera (2018)] etc.

Standard interval arithmetic's (IA) underlying assumption about the independency of all the operands is responsible for its overestimation problem. In this regard, affine arithmetic (AA) proves itself as an efficient tool to handle the overestimation problem and results with comparatively tighter enclosures. The concept of AA and its application in computer graphics are firstly introduced by Comba et al. [Comba and Stolfi (1993)]. After a few year, Stolfi et al. [Stolfi and De Figueiredo (2003)] illustrated the overestimation problem in case of standard IA and how AA is able to overcome it. Further, the concepts, properties and several applications of AA have been discussed by De Figueiredo et al. [De Figueiredo and Stolfi (2004)]. Miyajima et al. [Miyajima and Kashiwagi (2004)] proposed a dividing method by utilizing the best multiplication in Akhmerov [Akhmerov (2005)] developed an interval-affine Gaussian algorithm for constrained systems. A direct method for solving parametric interval linear systems with non-affine dependencies is demonstrated by Skalna [Skalna (2009)]. Rump et al. [Rump and Kashiwagi (2015)] discussed the improvements and implementations of Skalna et al. [Skalna and Hladík (2017)] developed a new algorithm for Chebyshev minimum-error multiplication of reduced affine forms. An optimization model based on improved affine arithmetic for interval power flow analysis has been developed by $\mathrm{Xu}$ et al. [Xu, Gu, Gao et al. (2016)]. Further, Adusumilli et al. [Adusumilli and Kumar (2018)] studied the 
modified affine arithmetic based continuation power flow analysis for voltage stability assessment under uncertainty. An affine arithmetic-based energy management system for isolated microgrids has been illustrated by Romero-Quete et al. [Romero-Quete and Cañizares (2018)]. Wang et al. [Wang, Wang, Teng et al. (2018)] discussed an affine arithmetic-based multi-objective optimization method for energy storage systems operating in active distribution networks with uncertainties.

There are many works of literature available for the solution of uncertain linear eigenvalue problems viz. uncertain SEP $(\tilde{A} \widetilde{x}=\tilde{\lambda} \widetilde{x})$ and uncertain GEP $(\tilde{A} \widetilde{x}=\tilde{\lambda} \widetilde{B} \widetilde{x})$. Hladik et al. [Hladik, Daney and Tsigaridas (2011)] proposed a filtering method for solving interval eigenvalue problems, which is based on the concept of sufficient conditions for singularity and regularity of interval matrices given by Rex et al. [Rex and Rohn (1998)]. The real eigenvalue bounds of linear (that is standard and generalized) real interval eigenvalue problems are studied by Leng [Leng (2014)]. Further, Mahato et al. [Mahato and Chakraverty (2016b)] extended the filtering algorithm given by Hladik et al. [Hladik, Daney and Tsigaridas (2011)] for eigenvalue bounds of fuzzy symmetric matrices to solve standard eigenvalue problem. Also, the filtering algorithm for real eigenvalue bounds of both interval and fuzzy GEPs are illustrated by Mahato et al. [Mahato and Chakraverty (2016a)]. To the best of our knowledge, there exist very few literature studies for the solution of uncertain (interval and/or fuzzy) NEPs. Chakraverty et al. [Chakraverty and Mahato (2018)] have been solved nonlinear interval eigenvalue problems for damped spring-mass systems using two methods viz. linear sufficient regularity perturbation (LSRP) method and direct sufficient regularity perturbation (DSRP) method. Further by using IA, Sadangi [Sadangi (2013)] solved interval NEPs.

This article is organized as follows. The present section of the article contains the introduction and literature survey. In Section 2, necessary preliminaries related to fuzzy set theory are discussed. These concepts are useful for the present investigation. Further, AA and its properties are included in Section 3. A fuzzy-affine approach (FAA) has been developed in Section 4. In Section 5, the general form of both crisp, as well as fuzzy nonlinear eigenvalue problem (FNEP) and the linearization procedure to convert it into GEP, are included. Then, the proposed method is explained in Section 6. Four illustrative numerical examples related to structural dynamics are investigated in Section 7, followed by concluding remarks in Section 8 .

\section{Preliminaries}

In this section, basic definitions related to present work viz. fuzzy set, fuzzy numbers and fuzzy arithmetic are illustrated [Dubois (1980); Kaufmann and Gupta (1988); Zimmermann (2001); Hanss (2005); Zadeh, Fu and Tanaka (2014)].

\subsection{Fuzzy set}

A fuzzy set is the set of ordered pairs and may be defined as

$\widetilde{F}=\left\{\left(x, \mu_{\widetilde{F}}(x)\right): x \in X, \mu_{\widetilde{F}}(x) \in[0,1]\right\}$,

where $\mu_{\widetilde{F}}(x)$ is known as the membership function of $\widetilde{F}$ and $X$ be the universal set. 


\subsection{Fuzzy number}

The fuzzy number $\widetilde{F}$ is defined as a special type of fuzzy set (given in Eq. (4)) that obeys the following properties:

1. $\widetilde{F}$ is normal, (that is $\exists x \in \mathbb{R}: \widetilde{F}(x)=1$ );

2. $\widetilde{F}$ is convex;

3. The membership function $\mu_{\widetilde{F}}(x)$ is piecewise continuous.

$\eta$-cut of a fuzzy number: Each $\eta$-cut $\widetilde{F}(\eta)$ of the fuzzy number $\widetilde{F}$, is a standard closed interval which depends upon the value of $\eta \in[0,1]$, and is represented as $\widetilde{F}(\eta)=[\underline{F(\eta)}, \overline{F(\eta)}]$.

Here $F(\eta)$ is the lower bound and $\overline{F(\eta)}$ is the upper bound of $\widetilde{F}(\eta)$. It may be noted that the fuzzy set $\widetilde{F}$ may be expressed uniquely and completely through the family of its $\eta$-cuts. Further, in the above second property, $\widetilde{F}$ is convex means all the $\eta$-cuts of $\widetilde{F}$, $\widetilde{F}(\eta)=\{x \in \mathbb{R} \mid \widetilde{F}(x) \geq \eta\}, \eta \in[0,1]$ are closed standard intervals.

There exist different types of fuzzy numbers viz. triangular fuzzy number (TFN), trapezoidal fuzzy number (TrFN), Gaussian fuzzy number (GFN) and exponential fuzzy number (EFN), etc. In our work mainly TFN and TrFN are considered.

\subsubsection{Triangular fuzzy number (TFN)}

TFN may be represented by a triplet $\widetilde{T}=\left(t_{1}, t_{2}, t_{3}\right)$. It generally has a linear graph (that is the composition of left-increasing and right-decreasing linear functions) as shown in Fig. 2. It may be noted that in TFN, there exists exactly one $x_{0} \in \mathbb{R}$ such that $\mu_{\widetilde{T}}\left(x_{0}\right)=1$. Here $x_{0}$ is known as the mean value of $\widetilde{T}$. The membership function $\mu_{\widetilde{T}}(x)$ of a TFN $\widetilde{T}=\left(t_{1}, t_{2}, t_{3}\right)$ may be defined as follows:

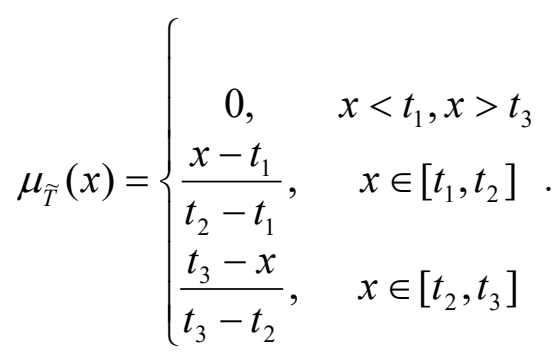




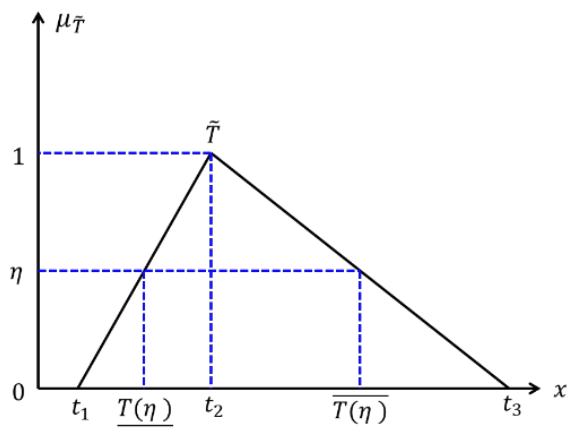

Figure 2: Triangular fuzzy number (TFN) $\widetilde{T}$

\subsubsection{Trapezoidal fuzzy number (TrFN)}

TrFN may be represented by a quadruplet $\widetilde{L}=\left(l_{1}, l_{2}, l_{3}, l_{4}\right)$. As shown in Fig. 3, there is an interval $x \in\left[l_{2}, l_{3}\right]$ such that $\mu_{\tilde{L}}(x)=1$. Thus, the membership function $\mu_{\tilde{L}}(x)$ may be defined as follows:

$\mu_{\widetilde{L}}(x)=\left\{\begin{array}{cc}0, & x<l_{1}, x>l_{4} \\ \frac{x-l_{1}}{l_{2}-l_{1}}, & x \in\left[l_{1}, l_{2}\right] \\ 1, & x \in\left[l_{2}, l_{3}\right] . \\ \frac{l_{4}-x}{l_{4}-l_{3}}, & x \in\left[l_{3}, l_{4}\right]\end{array}\right.$

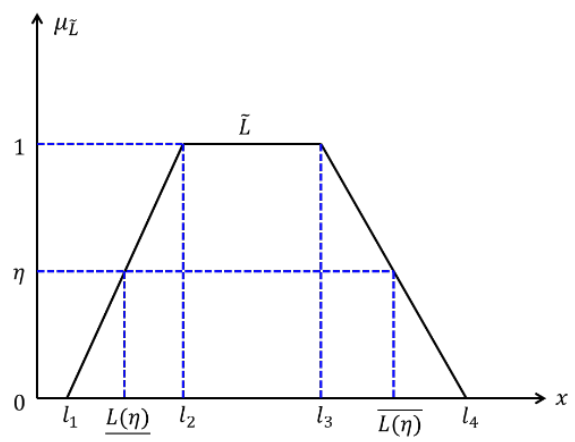

Figure 3: Trapezoidal fuzzy number $(\operatorname{TrFN}) \widetilde{L}$

\subsection{Fuzzy matrix}

A matrix $[\tilde{N}]=\left(\widetilde{n}_{p q}\right)$ of order $m \times n$ may be referred as fuzzy matrix, if each element $\left(\widetilde{n}_{p q}\right)$ for $p=1,2, \cdots, m$ and $q=1,2, \cdots, n$ is a fuzzy number. In this article, TFN and TrFN are particularly used. Further, a fuzzy matrix may be considered as non-negative $([\tilde{N}] \geq 0)$, if each element of the fuzzy matrix is a non-negative fuzzy number. 


\subsection{Parametric form of fuzzy number}

Any fuzzy number can be parameterized by using $\eta$-cut. Thus, $\widetilde{F}(\eta)$ is the parametric form of the fuzzy number $\widetilde{F}$ represented as $\widetilde{F}(\eta)=[\underline{F(\eta)}, \overline{F(\eta)}]$ for $\eta \in[0,1]$. It may be noted that lower and upper bounds of the parametric form of fuzzy number must have the following properties:

1. The lower bound $F(\eta)$ is a left bounded continuous non-decreasing function over $[0,1]$.

2. The upper bound $\overline{F(\eta)}$ is a right bounded continuous non-increasing function over $[0,1]$.

3. $\quad \underline{F(\eta)} \leq \overline{F(\eta)}$ for $\eta \in[0,1]$.

Firstly, the TFN $\widetilde{T}=\left(t_{1}, t_{2}, t_{3}\right)$ is converted into its parametric form by $\eta$-cut approach as $\tilde{T}(\eta)=[\underline{T(\eta)}, \overline{T(\eta)}]=\left[t_{1}+\eta\left(t_{2}-t_{1}\right), t_{3}-\eta\left(t_{3}-t_{2}\right)\right]$ where $\eta \in[0,1]$.

Next, the $\operatorname{TrFN} \widetilde{L}=\left(l_{1}, l_{2}, l_{3}, l_{4}\right)$ also transformed into its parametric form as

$\tilde{L}(\eta)=[L(\eta), \overline{L(\eta)}]=\left[l_{1}+\eta\left(l_{2}-l_{1}\right), l_{4}-\eta\left(l_{4}-l_{3}\right)\right]$, where $\eta \in[0,1]$.

In this regard, some of the terminologies of a fuzzy number $\widetilde{F}(\eta)=[\underline{F(\eta)}, \overline{F(\eta)}]$ through its parametric form may be described as follows:

Center: $\widetilde{F}_{c}=\frac{1}{2}\{\underline{F(\eta)}+\overline{F(\eta)}\}$.

Width: $\widetilde{F}_{w}=\overline{F(\eta)}-\underline{F(\eta)}$.

Radius: $\widetilde{F}_{\Delta}=\frac{1}{2}\{\overline{F(\eta)}-\underline{F(\eta)}\}$.

\subsection{Fuzzy arithmetic (FA)}

As mentioned in Section 2.4, any fuzzy number can be regarded as a family of standard intervals by using the parametric form ( $\eta$-cut). Let us consider two fuzzy numbers in its parametric representations as $\widetilde{S}=[\underline{S(\eta)}, \overline{S(\eta)}]$ and $\widetilde{T}=[\underline{T(\eta)}, \overline{T(\eta)}]$. Therefore, the standard interval-based FA may be illustrated as

$\widetilde{S} \otimes \widetilde{T}=\{S \otimes T: S \in S(\eta), T \in T(\eta)$ for $\eta \in[0,1]\}$,

where ' $\otimes$ ' stands for all the binary operations viz. $\{+,-,,, /\}$. In this regard, all the FA is discussed as follows:

Addition: $\widetilde{S}+\widetilde{T}=[\underline{S(\eta)}+\underline{T(\eta)}, \overline{S(\eta)}+\overline{T(\eta)}]$; 
Subtraction: $\widetilde{S}-\widetilde{T}=[\underline{S(\eta)}-\overline{T(\eta)}, \overline{S(\eta)}-\underline{T(\eta)}]$;

Scalar multiplication: $\alpha \cdot \widetilde{S}=\left\{\begin{array}{ll}{[\alpha \cdot S(\eta), \alpha \cdot \overline{S(\eta)}],} & \alpha \geq 0 \\ {[\alpha \cdot \overline{S(\eta)}, \alpha \cdot \underline{S(\eta)}]} & \alpha<0\end{array} ;\right.$

Multiplication: $\widetilde{S} \cdot \widetilde{T}=[\min \{Z(\widetilde{S}, \widetilde{T})\}, \max \{Z(\widetilde{S}, \widetilde{T})\}]$,

where $Z(\widetilde{S}, \widetilde{T})=\{\underline{S(\eta)} \cdot \underline{T(\eta)}, \underline{S(\eta)} \cdot \overline{T(\eta)}, \overline{S(\eta)} \cdot \underline{T(\eta)}, \overline{S(\eta)} \cdot \overline{T(\eta)}$;

Reciprocal: $\frac{1}{\widetilde{S}}=\left\{\begin{array}{cc}{\left[\frac{1}{\overline{S(\eta)}, \frac{1}{S(\eta)}}\right],} & 0 \notin \widetilde{S} \\ (-\infty, \infty), & 0 \in \widetilde{S}\end{array} ;\right.$

Division: $\frac{\widetilde{S}}{\widetilde{T}}=\widetilde{S} \cdot \frac{1}{\widetilde{T}}=\left\{\begin{array}{cc}\frac{[S(\eta), \overline{S(\eta)}] \cdot\left[\frac{1}{\overline{T(\eta)}}, \frac{1}{T(\eta)}\right]}{0 \notin \widetilde{S}} \\ (-\infty, \infty) & 0 \in \widetilde{S}\end{array} ;\right.$

Power:

- $\quad$ If $n>0$ is an odd integer, then $(\widetilde{S})^{n}=\left|S(\eta)^{n}, \overline{S(\eta)^{n}}\right|$;

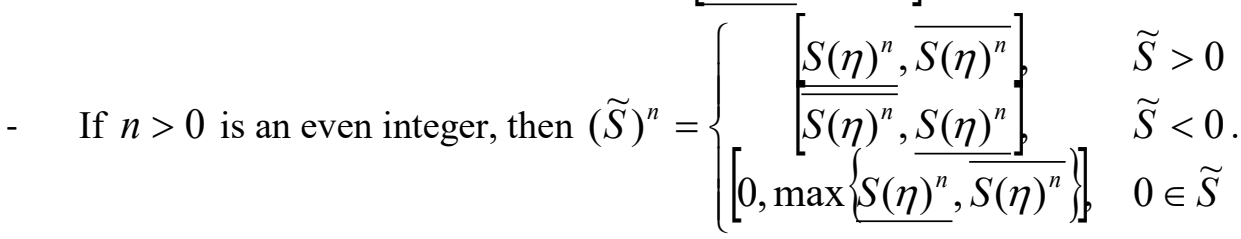

\section{Affine arithmetic (AA)}

One of the main obstacles in the wide-spread use of interval arithmetic (IA) in numerical calculations is that the range estimates computed with standard IA tend to be too large, especially in complicated expressions or long iterative computations. This is mainly due to the interval overestimation problem [De Figueiredo and Stolfi (2004); Skalna (2009)]. This overestimation problem is mainly because of IA's underlying assumption that the variables of the arguments to primitive operations may vary independently over their given intervals. In this regard, affine arithmetic (AA) [De Figueiredo and Stolfi (2004)] is a model for self-validated numerical computation that aims to attack the interval overestimation problem in standard IA. AA keeps track of first-order correlations between computed and input quantities. These correlations are automatically exploited in primitive operations, with the result that in many cases AA is able to produce interval estimates that are much better than the ones obtained with standard IA. Also, AA provides a geometric representation for the joint range of related quantities that can be exploited to increase the efficacy of interval methods. 


\subsection{Affine representation}

Affine form representation is a linear polynomial of real variables $\varepsilon_{i}$ known as noise symbols and these symbols lie in a particular interval $\mathbb{X}=[-1,1]$. Suppose, $\hat{S}$ denotes the affine form representation of an ideal quantity $S \in S_{0}+\left[-\sum_{i=1}^{p}\left|S_{i}\right|, \sum_{i=1}^{p}\left|S_{i}\right|\right]$. Then it may be expressed as

$S \in \hat{S}=S_{0}+\sum_{i=1}^{p} S_{i} \varepsilon_{i}=S_{0}+S_{1} \varepsilon_{1}+\cdots+S_{p-1} \varepsilon_{p-1}+S_{p} \varepsilon_{p}$,

where $\varepsilon_{i} \in \mathbb{X}=[-1,1]$ for $i=1,2, \cdots, p$. Here, the initial term $S_{0}$ is known as the central value of $\hat{S}$ and each associate coefficient $S_{i}$ for $i=1,2, \cdots, p$ of the respective noise symbol $\varepsilon_{i}$ is called the partial deviation of $\hat{S}$. These partial deviations are finite real numbers.

Further, all the noise symbols of an affine form representation are unique and independent to each other, whereas in two different affine form representations there may exist some common noise symbols. The number of noise symbols for different representations may not be equal. New noise symbols may also be generated during affine computations.

\subsection{Conversion between $A A$ and $I A$}

Suppose $\hat{S}$ is an affine form representation of the ideal quantity $S$ given as follows:

$\hat{S}=S_{0}+\sum_{i=1}^{p} S_{i} \varepsilon_{i}=S_{0}+S_{1} \varepsilon_{1}+\cdots+S_{p} \varepsilon_{p}$.

The total deviation of the above affine form representation may be illustrated as

$D_{S}=\sum_{i=1}^{p}\left|S_{i}\right|=\left|S_{1}\right|+\left|S_{2}\right|+\cdots+\left|S_{p}\right|$.

Thus the interval bounds $[S]$ of the affine form representation $\hat{S}$ may be computed as

$[S]=\left[S_{0}-D_{S}, S_{0}+D_{S}\right]$.

It may be noted that for every noise symbol ranges over the interval $\mathbb{X}=[-1,1]$ independently, $[S]$ is the smallest interval that contains all the possible values of $\hat{S}$. After $\hat{S}$ is converted into $[S]$, all the presented correlation information in the affine form is discarded.

Conversely,

The affine form representation of the interval $[S]=[\underline{S}, \bar{S}]$ may be calculated as

$\hat{S}=S_{0}+S_{q} \varepsilon_{q}$, 
where $S_{0}=\frac{1}{2}(\underline{S}+\bar{S})$ is the center and $S_{q}=\frac{1}{2}(\bar{S}-\underline{S})$ is the radius of the given interval $[S]$. Further, $\varepsilon_{q} \in \mathbb{X}=[-1,1]$ is the newly generated noise symbol that should not be present in any other existing affine representations.

\subsection{Affine arithmetic operations}

Let us consider two affine representations $\hat{S}$ and $\hat{T}$ such that, $\hat{S}=S_{0}+\sum_{i=1}^{p} S_{i} \varepsilon_{i}$ and $\hat{T}=T_{0}+\sum_{i=1}^{p} T_{i} \varepsilon_{i}$.

Then, the AA operations the affine representations may be defined as follows [De Figueiredo and Stolfi (2004)]:

Addition: $\hat{S}+\hat{T}=\left(S_{0}+T_{0}\right)+\sum_{i=1}^{p}\left(S_{i}+T_{i}\right) \varepsilon_{i}$;

Subtraction: $\hat{S}-\hat{T}=\left(S_{0}-T_{0}\right)+\sum_{i=1}^{p}\left(S_{i}-T_{i}\right) \varepsilon_{i}$;

Scalar multiplication: $\alpha \cdot \hat{S}=\alpha \cdot S_{0}+\sum_{i=1}^{p}\left(\alpha \cdot S_{i}\right) \varepsilon_{i}$, where $\alpha \in \mathbb{R}$;

Multiplication: $\hat{S} \cdot \hat{T}=S_{0} T_{0}+\sum_{i=1}^{p}\left(S_{0} T_{i}+S_{i} T_{0}\right) \varepsilon_{i}+\kappa_{q} \varepsilon_{q}$,

where

$\left|\kappa_{q}\right| \geq\left|\sum_{i=1}^{P} S_{i} \varepsilon_{i} \cdot \sum_{i=1}^{p} T_{i} \varepsilon_{i}\right|, \varepsilon_{i} \in \mathbb{X}=[-1,1]$.

Here, $\varepsilon_{q}$ is the newly generated noise symbol during multiplication and $\left|\kappa_{q}\right|$ is the upper bound of the approximation error.

Division: $\frac{\hat{S}}{\hat{T}}=\hat{S} \cdot \frac{1}{\hat{T}}=\frac{S_{0}}{T_{0}}+\frac{1}{\hat{T}} \sum_{i=1}^{p}\left(S_{i}-\frac{S_{0}}{T_{0}} T_{i}\right) \varepsilon_{i}$, provided $\hat{S} \neq\{0\} \quad$ [Skalna (2009)].

To prove that AA is an efficient tool to overcome the "interval overestimation problem", the following example of an interval nonlinear function is considered.

Example 1: Let us consider a nonlinear function such that

$f(S, T)=S^{2}+2 T^{2}-2 S T$,

where $\forall S \in[1,3]$ and $\forall T \in[5,7]$.

Applying standard interval computations, the following result is obtained.

$$
\begin{array}{r}
{[f]([S],[T])=[S]^{2}+2[T]^{2}-2[S][T]} \\
=[9,97]
\end{array}
$$

Therefore, for standard IA $f(S, T) \in[9,97]$. 
Further, the interval bounds of the present variables are converted into its affine form representations as follows:

$$
\begin{aligned}
& {[S]=[1,3] \Rightarrow \hat{S}=2+\varepsilon_{1}} \\
& {[T]=[5,7] \Rightarrow \hat{T}=6+\varepsilon_{2}}
\end{aligned}
$$

where $\varepsilon_{1}$ and $\varepsilon_{2}$ are the noise symbols of the affine form representations and $\varepsilon_{i} \in[-1,1]$ for $i=1,2$. Then by using AA operations, the affine form of the nonlinear function $f(S, T)$ may be evaluated as follows:

$$
\begin{aligned}
\hat{f}(\hat{S}, \hat{T})=\hat{S}^{2}+2 \hat{T}^{2} & -2 \hat{S} \hat{T} \\
& =52-8 \varepsilon_{1}+20 \varepsilon_{2}+\varepsilon_{3}+2 \varepsilon_{4}-2 \varepsilon_{5}
\end{aligned}
$$

where $\varepsilon_{i} \in[-1,1]$ for $i=3,4,5$ are newly generated noise symbols during the affine operation to evaluate the required result.

Hence after converting the resulting affine solution into its interval bounds using Eq. (24), we may have

$\hat{f}(\hat{S}, \hat{T})=[52-33,52+33]=[19,85]$.

Since the central value and the total deviation of the affine function $\hat{f}$ are $f_{0}=52$ and $d_{f}=33$ respectively. Therefore, it may be clearly noticed from Eq. (32) and Eq. (35) that

$$
f(S, T) \in \hat{f}(\hat{S}, \hat{T}) \subset[f]([S],[T]) .
$$

The present example shows that AA may result in better enclosures as compare to the standard IA. Moreover, Skalna et al. [Skalna and Hladík (2017)] developed an algorithm for Chebyshev minimum-error multiplication of reduced affine forms which will result in the interval $[23.5,85]$. Thus minimum-error Chebyshev multiplication of affine forms is indeed a good approximation than that of AA. Hence, the proposed procedure may be extended by using Chebyshev minimum-error multiplication instead of standard AA multiplication (given in Eq. (29)) for better results.

\section{Fuzzy-affine form}

From Section 2.4, a fuzzy number may be regarded as a family of intervals by parameterized by using $\eta$-cut method. That interval further may be transformed into an affine form representation to handle the fuzzy number more efficiently.

Now, let us assume a TFN $\widetilde{T}=\left(t_{1}, t_{2}, t_{3}\right)$. Parameterizing it using $\eta$-cut as mentioned in Eq. (8), we may have

$$
\widetilde{T}(\eta)=[\underline{T(\eta)}, \overline{T(\eta)}]=\left[t_{1}+\eta\left(t_{2}-t_{1}\right), t_{3}-\eta\left(t_{3}-t_{2}\right)\right] \text { for } \eta \in[0,1] \text {. }
$$

Further, the above interval quantity may be transformed into its affine form representation (Eq. (25)) as 
$\hat{T}\left(\eta, \varepsilon_{T}\right)=\frac{1}{2}\left\{\left(t_{1}+t_{3}\right)+\eta\left(2 t_{2}-t_{1}-t_{3}\right)\right\}+\frac{1}{2}\left\{\left(t_{3}-t_{1}\right)+\eta\left(t_{1}-t_{3}\right)\right\} \varepsilon_{T}$,

for $\eta \in[0,1]$ and $\varepsilon_{T} \in[-1,1]$.

In the similar fashion, a $\operatorname{TrFN} \widetilde{L}=\left(l_{1}, l_{2}, l_{3}, l_{4}\right)$ may be converted into its fuzzy-affine form using Eq. (25) as follows:

$\hat{L}\left(\eta, \varepsilon_{L}\right)=\frac{1}{2}\left\{\left(l_{1}+l_{4}\right)+\eta\left(\left(l_{2}+l_{3}\right)-\left(l_{1}-l_{4}\right)\right)\right\}+\frac{1}{2}\left\{\left(l_{4}-l_{1}\right)+\eta\left(\left(l_{1}+l_{3}\right)-\left(l_{2}+l_{4}\right)\right)\right\} \varepsilon_{L},(38$

for $\eta \in[0,1]$ and $\varepsilon_{L} \in[-1,1]$.

Let us consider the fuzzy-affine forms $\hat{S}\left(\eta, \varepsilon_{S}\right)$ and $\hat{T}\left(\eta, \varepsilon_{T}\right)$ of two fuzzy numbers $\widetilde{S}$ and $\widetilde{T}$ (where $\eta \in[0,1]$ and $\varepsilon_{S}, \varepsilon_{T} \in[-1,1]$ ) respectively. Then all the arithmetic for fuzzy-affine numbers can be obtained as

$\hat{R}\left(\eta, \varepsilon_{S}, \varepsilon_{T}\right)=\hat{S}\left(\eta, \varepsilon_{S}\right) \otimes \hat{T}\left(\eta, \varepsilon_{T}\right)$.

Therefore, the resulting solution may be computed as

$\widetilde{S} * \widetilde{T}=\left[\min _{\varepsilon_{S}, \varepsilon_{T} \in[-1,1]} \hat{R}\left(\eta, \varepsilon_{S}, \varepsilon_{T}\right), \max _{\varepsilon_{S}, \varepsilon_{T} \in[-1,1]} \hat{R}\left(\eta, \varepsilon_{S}, \varepsilon_{T}\right)\right]$ for $\forall \eta \in[0,1]$.

Example 2: Let us consider the nonlinear function $f(S, T)=S^{2}+2 T^{2}-2 S T$ that given in Eq. (31) of Example 1, in which the consisting variables are taken in the form of TFNs such as

$\forall S \in \widetilde{S}=(1,2,3)$ and $\forall T \in \widetilde{T}=(5,6,7)$.

Then after parameterizing the above TFNs (41a) and (41b) as given in Section 2.4, we may have

$\widetilde{S}(\eta)=[1+\eta, 3-\eta]$ and $\widetilde{T}(\eta)=[5+\eta, 7-\eta]$ for $\eta \in[0,1]$.

Applying basic FA given in Section 2.5, the following result is obtained.

$\widetilde{f}\left(\tilde{S}(\eta), \widetilde{T}(\eta)=\left[\eta^{2}+42 \eta+9, \eta^{2}-46 \eta+97\right]\right.$

Further, the fuzzy-affine forms of $\widetilde{S}$ and $\widetilde{T}$ are found below.

$\hat{S}\left(\eta, \varepsilon_{1}\right)=2+(1-\eta) \varepsilon_{1}$ and $\hat{T}\left(\eta, \varepsilon_{2}\right)=6+(1-\eta) \varepsilon_{2}$,

where $\eta \in[0,1]$ and $\varepsilon_{i}=[-1,1]$ for $i=1,2$ are the noise symbols of the fuzzy-affine forms.

Then by using FAA, the required fuzzy-affine form of the given nonlinear function $f(S, T)$ is obtained as follows:

$\hat{f}\left(\hat{S}\left(\eta, \varepsilon_{1}\right), \hat{T}\left(\eta, \varepsilon_{2}\right)\right)=52-8(1-\eta) \varepsilon_{1}+20(1-\eta) \varepsilon_{2}+(1-\eta)^{2} \varepsilon_{3}+2(1-\eta)^{2} \varepsilon_{4}-2(1-\eta)^{2} \varepsilon_{5}$ 
where $\varepsilon_{i} \in[-1,1]$ for $i=3,4,5$ are newly generated noise symbols during FAA.

Corresponding fuzzy (TFN) functional value of the nonlinear function $f(S, T)$ is plotted in Fig. 2. In the figure, the lines marked with ' $\square$ ' depict the FAA and the line marked with '*' stand for FA. From the plot, it may be noted that FAA is more efficient and results in better enclosures than basic FA with respect to the considered example.

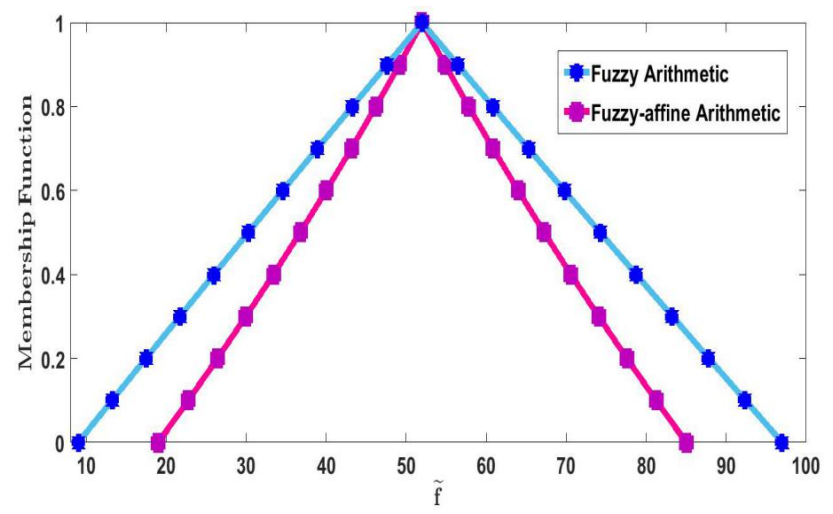

Figure 2: Comparison plot between fuzzy arithmetic (FA) and fuzzy-affine approach (FAA) Further, Tab. 1 contains the fuzzy functional values of the given nonlinear function for $\eta=0,0.1,0.3,0.6,0.8,1$. Therefore, it may be seen from Tab. 1 that the FAA yields tighter outer bounds to the functional value as compared with standard FA.

Table 1: Comparison table between FA and FAA for some values of $\eta$

\begin{tabular}{ccccc}
\hline \multirow{2}{*}{ Iterations } & \multicolumn{2}{c}{ Fuzzy arithmetic (FA) } & \multicolumn{2}{c}{ Fuzzy-affine approach (FAA) } \\
\cline { 2 - 4 } & Lower bound $(\underline{f})$ & Upper bound $(\bar{f})$ & Lower bound $(\underline{f})$ & Upper bound $(\bar{f})$ \\
\hline$\eta=0$ & 9.0000 & 97.0000 & 19.0000 & 85.0000 \\
\hline$\eta=0.1$ & 13.2100 & 92.4100 & 22.7500 & 81.2500 \\
\hline$\eta=0.6$ & 21.6900 & 83.2900 & 29.9500 & 74.0500 \\
\hline$\eta=0.8$ & 34.5600 & 69.7600 & 40.0000 & 64.0000 \\
\hline$\eta=1$ & 43.2400 & 60.8400 & 46.2000 & 57.8000 \\
$(f=\bar{f})$ & 52.0000 & 52.0000 & 52.0000 & 52.0000 \\
\hline
\end{tabular}

It may be clearly noticed that for Example 2, FAA results in better and tighter enclosures as compared to basic FA.

\section{Nonlinear eigenvalue problem (NEP)}

In this section, general definitions and notations about NEP are discussed. Further, the linearization procedure [Tisseur and Meerbergen (2001); Mehrmann and Watkins (2002)] of NEP having degree ' $m$ ' into GEP has been illustrated here. 


\subsection{Nonlinear eigenvalue problem}

The NEP having parameters in the form of crisp numbers is defined as

$N(\lambda) x=0$,

where $\lambda$ is the crisp eigenvalue of and $x$ is the eigenvector corresponding to the eigenvalue $\lambda$ of the above problem. Further, $N(\lambda)$ is the matrix-valued nonlinear function of $\lambda$. The general form of the above NEP having degree ' $m$ ' may be illustrated as follows:

$N(\lambda) x=\sum_{j=0}^{m} \lambda^{j} N_{j} x=\left(N_{0}+N_{1} \lambda+\cdots \cdots+N_{m-1} \lambda^{m-1}+N_{m} \lambda^{m}\right) x=0$,

where the coefficients $N_{j}$ for $j=0,1, \cdots, m$ are crisp square matrices.

It may be noted that the linear eigenvalue problem is a special case with $m=1$,

$N(\lambda)=N_{0}-\lambda N_{1}$.

For $N_{0}=A$ and $N_{1}=B$, Eq. (48) is known as GEP whereas for $N_{0}=A$ and $N_{1}=I$ (' $I$ ' stands for the identity matrix) it is known as a standard eigenvalue problem.

Moreover, depending upon the degree of $\lambda$, there exist different forms of NEPs such as quadratic eigenvalue problem (QEP) $\left(N(\lambda) x=\left(N_{0}+N_{1} \lambda+N_{2} \lambda^{2}\right) x=0\right)$ and cubic eigenvalue problem (CEP) $\left(N(\lambda) x=\left(N_{0}+N_{1} \lambda+N_{2} \lambda^{2}+N_{3} \lambda^{3}\right) x=0\right)$, etc.

\subsection{Fuzzy nonlinear eigenvalue problem (FNEP)}

The case where uncertain parameters are considered in the form of fuzzy numbers, the NEP may be referred as FNEP and is denoted as follows:

$\tilde{N}(\tilde{\lambda}) \tilde{x}=0$,

where $\tilde{\lambda}$ is the fuzzy eigenvalue, $\widetilde{x}$ is the corresponding fuzzy eigenvector and $\tilde{N}(\tilde{\lambda})$ is the fuzzy matrix-valued nonlinear function in $\tilde{\lambda}$. The general form of the FNEP of degree ' $m$ ' may be considered in the following form.

$$
\tilde{N}(\tilde{\lambda}) \tilde{x}=\sum_{j=0}^{m} \tilde{\lambda}^{j} \tilde{N}_{j} \tilde{x}=\left(\tilde{N}_{0}+\tilde{N}_{1} \tilde{\lambda}+\cdots \cdots+\widetilde{N}_{m-1} \tilde{\lambda}^{m-1}+\tilde{N}_{m} \tilde{\lambda}^{m}\right) \tilde{x}=0,
$$

where each of the coefficients $\tilde{N}_{j}$ for $j=0,1, \cdots, m$ are fuzzy square matrices of order $n \times n$ having elements $\left(\widetilde{n}_{j}\right)_{p q}$ for $p, q=1,2, \cdots, n$ in the form of either TFNs or TrFNs.

In the similar manner as given for crisp quantities in Section 5.1, fuzzy QEP

$$
\left(\tilde{N}(\tilde{\lambda}) \tilde{x}=\left(\tilde{N}_{0}+\tilde{N}_{1} \tilde{\lambda}+\tilde{N}_{2} \widetilde{\lambda}^{2}\right) \tilde{x}=0\right)
$$

and fuzzy CEP $\left(\tilde{N}(\tilde{\lambda}) \tilde{x}=\left(\tilde{N}_{0}+\tilde{N}_{1} \tilde{\lambda}+\tilde{N}_{2} \tilde{\lambda}^{2}+\tilde{N}_{3} \tilde{\lambda}^{3}\right) x=0\right)$, may also be defined. 


\subsection{Linearization of nonlinear eigenvalue problem}

Let us consider the general form of NEP given by Eq. (47) having degree $m$ as follows:

$$
\begin{aligned}
& N(\lambda) x=\sum_{j=0}^{m} \lambda^{j} N_{j} x=\left(N_{0}+N_{1} \lambda+\cdots \cdots+N_{m-1} \lambda^{m-1}+N_{m} \lambda^{m}\right) x=0, \\
& \Rightarrow N_{0} x+N_{1} \lambda x+\cdots \cdots+N_{m-1} \lambda^{m-1} x+N_{m} \lambda^{m} x=0 .
\end{aligned}
$$

We now define new variables $x_{j}$ for $j=1,2, \cdots, m$ such that,

$x_{1}:=x$ and $x_{j+1}:=\lambda x_{j}=\lambda^{j} x$ for $j=1,2, \cdots, m-1$

Therefore substituting Eq. (52) in Eq. (51), the given NEP $N(\lambda) x=0$ may get transformed into a linear eigenvalue problem as follows:

$N_{0} x_{1}+N_{1} \lambda x_{1}+N_{2} \lambda x_{2}+\cdots \cdots+N_{m-1} \lambda x_{m-1}+N_{m} \lambda x_{m}=0$.

Further the above system in Eq. (53) can be represented in the following matrix form [Mehrmann and Watkins (2002)].

$$
\left(\begin{array}{c|ccc}
-N_{0} & 0 & \cdots & 0 \\
\hline 0 & I & & 0 \\
\vdots & \vdots & \ddots & \vdots \\
0 & 0 & & I
\end{array}\right) \cdot\left[\begin{array}{c}
x_{1} \\
\hline x_{2} \\
\vdots \\
x_{m}
\end{array}\right]=\lambda \cdot\left(\begin{array}{ccc|c}
N_{1} & \cdots & N_{m-1} & N_{m} \\
\hline I & & 0 & 0 \\
\vdots & \ddots & \vdots & \vdots \\
0 & & I & 0
\end{array}\right) \cdot\left[\begin{array}{c}
x_{1} \\
\vdots \\
x_{m-1} \\
\hline x_{m}
\end{array}\right] .
$$

The above system yields the GEP $A x^{*}=\lambda B x^{*}$, where $(A-\lambda B) \in \mathbb{C}^{m n, m n}$.

It may be noted that the NEP $N(\lambda) x=0$ has the same eigenvalues as the newly formed GEP $A x^{*}=\lambda B x^{*}$ with the eigenvectors

$\left(x^{*}\right)^{T}=\left[x_{1}{ }^{T}, x_{2}{ }^{T}, \cdots, x_{m}{ }^{T}\right]^{T}=\left[x^{T}, \lambda x^{T}, \cdots, \lambda^{m-1} x^{T}\right]^{T}$.

The NEP $N(\lambda) x=0$ of degree ' $m$ ' (whose coefficient matrices is of order $n \times n$ ) has ' $m n$ ' number of eigenvalues because after linearization the coefficient matrices of the newly formed GEP are of order $m n \times m n$.

\section{Proposed method for FNEP}

Let us consider the FNEP (Eq. (50)) of degree ' $m$ ' as follows:

$$
\tilde{N}(\tilde{\lambda}) \tilde{x}=\sum_{j=0}^{m} \tilde{\lambda}^{j} \tilde{N}_{j} \tilde{x}=\left(\tilde{N}_{0}+\tilde{N}_{1} \tilde{\lambda}+\cdots \cdots+\tilde{N}_{m-1} \tilde{\lambda}^{m-1}+\tilde{N}_{m} \tilde{\lambda}^{m}\right) \tilde{x}=0,
$$

where all the coefficient matrices $\widetilde{N}_{j}$ for $j=0,1, \cdots, m$ are fuzzy matrices of order $n \times n$ having elements $\left(\widetilde{n}_{j}\right)_{p q}$ for $p, q=1,2, \cdots, n$ in the form of either TFNs or TrFNs. Thus, the fuzzy coefficient matrices may be written as 


$$
\widetilde{N}_{j}=\left(\begin{array}{ccccc}
\left(\widetilde{n}_{j}\right)_{11} & \left(\widetilde{n}_{j}\right)_{12} & \cdots & \cdots & \left(\widetilde{n}_{j}\right)_{1 n} \\
\left(\widetilde{n}_{j}\right)_{21} & \left(\widetilde{n}_{j}\right)_{22} & \cdots & \cdots & \left(\widetilde{n}_{j}\right)_{2 n} \\
\vdots & \vdots & \ddots & & \vdots \\
\vdots & \vdots & & \ddots & \vdots \\
\left(\widetilde{n}_{j}\right)_{n 1} & \left(\widetilde{n}_{j}\right)_{n 2} & \cdots & \cdots & \left(\widetilde{n}_{j}\right)_{n n}
\end{array}\right) \text {, for } j=0,1, \cdots, m
$$

Adopting the parameterization of each fuzzy number as given in Section 2.4 (by using $\eta$ cut), the FNEP Eq. (55) in is converted into fuzzy parametric NEP.

$$
\tilde{N}(\tilde{\lambda}(\eta)) \widetilde{x}(\eta)=\sum_{j=0}^{m}\left\{\widetilde{\lambda}^{j}(\eta) \widetilde{N}_{j}(\eta)\right\} \widetilde{x}(\eta)=\left\{\widetilde{N}_{0}(\eta)+\widetilde{N}_{1}(\eta) \tilde{\lambda}(\eta)+\cdots \cdots+\widetilde{N}_{m}(\eta) \widetilde{\lambda}^{m}(\eta)\right\} \widetilde{x}(\eta)=0,
$$

where the fuzzy parametric coefficient matrices may be written as

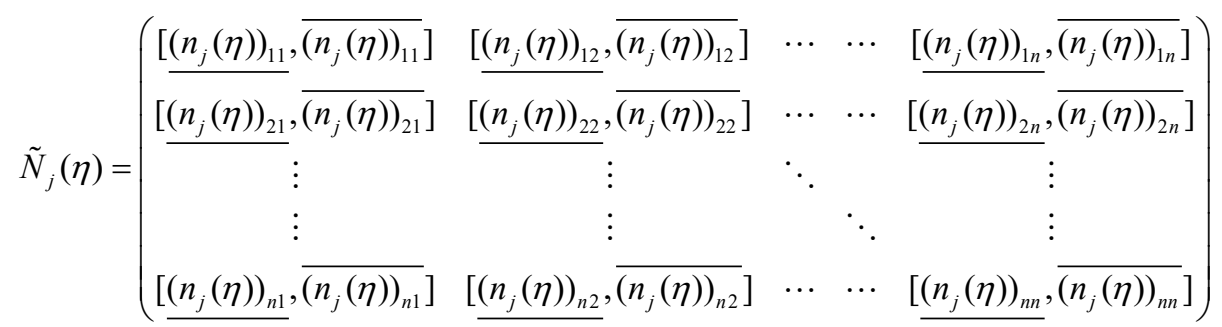

for $\eta \in[0,1]$.

Further, the fuzzy parametric NEP is again converted into its affine form representation as given in Section 4. Thus, the fuzzy-affine NEP of degree $m$ may be written as

$$
\begin{aligned}
& \hat{N}\left(\eta, \varepsilon^{*}\right)\left(\hat{\lambda}\left(\eta, \varepsilon^{*}\right)\right) \hat{x}\left(\eta, \varepsilon^{*}\right)=\sum_{j=0}^{m}\left\{\tilde{\lambda}^{j}\left(\eta, \varepsilon^{*}\right) \widetilde{N}_{j}\left(\eta, \varepsilon_{j, p q}\right)\right\} \widetilde{x}\left(\eta, \varepsilon^{*}\right)=0 \\
& \Rightarrow\left\{\widetilde{N}_{0}\left(\eta, \varepsilon_{0, p q}\right)+\widetilde{N}_{1}\left(\eta, \varepsilon_{1, p q}\right) \tilde{\lambda}\left(\eta, \varepsilon^{*}\right)+\cdots \cdots+\widetilde{N}_{m}\left(\eta, \varepsilon_{m, p q}\right) \widetilde{\lambda}^{m}\left(\eta, \varepsilon^{*}\right)\right\} \widetilde{x}\left(\eta, \varepsilon^{*}\right)=0
\end{aligned}
$$

where $\varepsilon^{*}$ may be either a newly generated noise symbol or a function existing noise symbols $\varepsilon_{j, p q}$. Here the coefficient matrices are in the form of fuzzy-affine representations given as

$$
\hat{N}_{j}\left(\eta, \varepsilon_{j, p q}\right)=\left(\begin{array}{ccccc}
\left(\widetilde{n}_{j}\left(\eta, \varepsilon_{j, 11}\right)\right)_{11} & \left(\widetilde{n}_{j}\left(\eta, \varepsilon_{j, 12}\right)\right)_{12} & \cdots & \cdots & \left(\widetilde{n}_{j}\left(\eta, \varepsilon_{j, 1 n}\right)\right)_{1 n} \\
\left(\widetilde{n}_{j}\left(\eta, \varepsilon_{j, 21}\right)\right)_{21} & \left(\widetilde{n}_{j}\left(\eta, \varepsilon_{j, 22}\right)\right)_{22} & \cdots & \cdots & \left(\widetilde{n}_{j}\left(\eta, \varepsilon_{j, 2 n}\right)\right)_{2 n} \\
\vdots & \vdots & \ddots & & \vdots \\
\vdots & \vdots & & \ddots & \vdots \\
\left(\widetilde{n}_{j}\left(\eta, \varepsilon_{j, n 1}\right)\right)_{n 1} & \left(\widetilde{n}_{j}\left(\eta, \varepsilon_{j, n 2}\right)\right)_{n 2} & \cdots & \cdots & \left(\widetilde{n}_{j}\left(\eta, \varepsilon_{j, n n}\right)\right)_{n n}
\end{array}\right) \text {, }
$$

where $\eta \in[0,1]$ and $\varepsilon_{j, p q} \in[-1,1]$ for $j=0,1, \cdots, m$ and $p, q=0,1, \cdots, n$. Moreover, $\hat{\lambda}\left(\eta, \varepsilon^{*}\right)$ and $\hat{x}\left(\eta, \varepsilon^{*}\right)$ are the corresponding fuzzy-affine forms of fuzzy eigenvalue $\tilde{\lambda}$ and fuzzy eigenvector $\tilde{x}$ of the FNEP respectively. 
Thus, the FNEP is transformed into a form involving several parameters such as $\eta$ and $\varepsilon^{*}$. Hence, now it can be linearized into GEP.

Utilizing the linearization procedure for the NEP given in Section 5.3, the fuzzy-affine NEP may be transformed into a fuzzy-affine GEP as

$\hat{A}\left(\eta, \varepsilon^{*}\right) \hat{x}^{*}\left(\eta, \varepsilon^{*}\right)=\hat{\lambda}\left(\eta, \varepsilon^{*}\right) \hat{B}\left(\eta, \varepsilon^{*}\right) \hat{x}^{*}\left(\eta, \varepsilon^{*}\right)$,

where

$$
\hat{A}=\left(\begin{array}{c|ccc}
-N_{0}\left(\eta, \varepsilon_{0, p q}\right) & 0 & \cdots & 0 \\
\hline 0 & I & & 0 \\
\vdots & \vdots & \ddots & \vdots \\
0 & 0 & & I
\end{array}\right)_{m n \times m n} \text { and }
$$

$$
\hat{B}=\left(\begin{array}{ccc|c}
N_{1}\left(\eta, \varepsilon_{1, p q}\right) & \cdots & N_{m-1}\left(\eta, \varepsilon_{m-1, p q}\right) & N_{m}\left(\eta, \varepsilon_{m, p q}\right) \\
\hline I & & 0 & 0 \\
\vdots & \ddots & \vdots & \vdots \\
0 & & I & 0
\end{array}\right)_{m n \times m n} .
$$

Further, the fuzzy-affine GEP (60) is converted to standard eigenvalue problem containing different parameters viz. $\eta$ and $\varepsilon^{*}$ given as follows:

$\hat{P}\left(\eta, \varepsilon^{*}\right) \hat{x}^{*}\left(\eta, \varepsilon^{*}\right)=\hat{\lambda}\left(\eta, \varepsilon^{*}\right) \hat{x}^{*}\left(\eta, \varepsilon^{*}\right)$,

where $\hat{P}\left(\eta, \varepsilon^{*}\right)=\left(\hat{B}\left(\eta, \varepsilon^{*}\right)\right)^{-1} * \hat{A}\left(\eta, \varepsilon^{*}\right)$.

The above standard eigenvalue problem (Eq. (61)) may be solved symbolically (by using MATLAB code "e=eig(A)") with symbols $\eta \in[0,1]$ and $\varepsilon_{j, p q} \in[-1,1]$ for $j=0,1, \cdots, m$ and $p, q=0,1, \cdots, n$. Thus, the required eigenvalue solutions in fuzzyaffine forms $\hat{\lambda}_{i}\left(\eta, \varepsilon^{*}\right)$ for $i=1,2, \cdots, m n$ may be computed.

Since every consisting noise symbols vary from -1 to 1 , the fuzzy parametric eigenvalues $\tilde{\lambda}_{i}(\eta)=\left[\underline{\lambda_{i}(\eta)}, \overline{\lambda_{i}(\eta)}\right]$ may be determined as follows:

$\underline{\lambda_{i}(\eta)}=\min _{\varepsilon^{*} \in[-1,1]} \hat{\lambda}_{i}\left(\eta, \varepsilon^{*}\right)$ and $\overline{\lambda_{i}(\eta)}=\max _{\varepsilon^{*} \in[-1,1]} \hat{\lambda}_{i}\left(\eta, \varepsilon^{*}\right)$, for $i=1,2, \cdots, m n$

Finally, all the fuzzy solutions of the FNEP of degree $m$ can be evaluated by varying $\eta$ from 0 to 1 .

\section{Numerical Examples}

To illustrate the applicability of the proposed method, five numerical examples have been solved in this section. Initially, a fuzzy QEP is considered having parameters in the form of TFNs. In the next problem, a damped spring-mass structural system (in which all the 
parameters are in the form of $\operatorname{TrFN}$ ) is solved by using the present approach. Further, in both third and fourth problems an application problem from structural dynamics is handled, which contains ' $n$ ' degrees-of-freedom connected damped spring-mass system. In the third one, the degree-of-freedom is taken as 3 and the parameters are in the form of TFN, while the forth problem contains TrFNs and has 5 degrees-of-freedom. Finally, a fuzzy CEP has worked out whose parameters are TFNs.

Example 3. Let us consider a fuzzy QEP (Eq. (50)) (crisp form given in Fazeli et al. [Fazeli and Rabiei (2016)]) $\tilde{N}(\tilde{\lambda}) \widetilde{x}=\left(\widetilde{N}_{0}+\widetilde{N}_{1} \tilde{\lambda}+\widetilde{N}_{2} \widetilde{\lambda}^{2}\right) \widetilde{x}=0$, where the coefficient matrices are considered as $4 \times 4$ fuzzy matrices (the elements are in TFNs) given as follows:

$$
\begin{aligned}
\tilde{N}_{0} & =\left[\begin{array}{llll}
-3.0475 & -2.1879 & -1.9449 & -2.8242 \\
-2.6500 & -2.4724 & -2.3515 & -2.1053 \\
-0.7456 & -0.6423 & -1.3117 & -0.1852 \\
-4.0500 & -3.0631 & -2.8121 & -3.7794
\end{array}\right], \\
\tilde{N}_{1} & =\left[\begin{array}{lllc}
(0.20,0.22,0.24) & (0.01,0.02,0.03) & (0.10,0.12,0.14) & (0.13,0.14,0.15) \\
(0.01,0.02,0.03) & (0.12,0.14,0.16) & (0.03,0.04,0.05) & (-0.08,-0.06,-0.04) \\
(0.10,0.12,0.14) & (0.03,0.04,0.05) & (0.24,0.28,0.32) & (0.04,0.08,0.12) \\
(0.13,0.14,0.15) & (-0.08,-0.06,-0.04) & (0.04,0.08,0.12) & (0.22,0.26,0.30)
\end{array}\right] \text { and } \\
\tilde{N}_{2} & =\left[\begin{array}{cccc}
(0.44,0.47,0.50) & (0.07,0.17,0.27) & (-0.35,-0.25,-0.15) & (0.50,0.54,0.58) \\
(-0.15,-0.11,-0.07) & (0.30,0.35,0.40) & (0.65,0.67,0.69) & (-0.34,-0.32,-0.30) \\
(0.50,0.55,0.60) & (0.40,0.43,0.46) & (0.30,0.36,0.42) & (-0.78,-0.74,-0.70) \\
1 & 1
\end{array}\right] .
\end{aligned}
$$

Here, particularly the $\widetilde{N}_{0}$ matrix is taken as a crisp matrix and the other two are fuzzy matrices having elements as TFNs. The above fuzzy matrices $\tilde{N}_{1}$ and $\widetilde{N}_{2}$ are parameterized by using $\eta$-cut approach given in Section 2.4 as follows:

$$
\begin{array}{r}
\tilde{N}_{1}(\eta)=\left[\begin{array}{cccc}
{[0.22+0.02 \eta, 0.24-0.02 \eta]} & {[0.01+0.01 \eta, 0.03-0.01 \eta]} & {[0.10+0.02 \eta, 0.14-0.02 \eta]} & {[0.13+0.01 \eta, 0.15-0.01 \eta]} \\
{[0.01+0.01 \eta, 0.03-0.01 \eta]} & {[0.12+0.02 \eta, 0.16-0.02 \eta]} & {[0.03+0.01 \eta, 0.05-0.01 \eta]} & {[-0.08+0.02 \eta,-0.04-0.02 \eta]} \\
{[0.10+0.02 \eta, 0.14-0.02 \eta]} & {[0.03+0.01 \eta, 0.05-0.01 \eta]} & {[0.24+0.04 \eta, 0.32-0.04 \eta]} & {[0.04+0.04 \eta, 0.12-0.04 \eta]} \\
{[0.13+0.01 \eta, 0.15-0.01 \eta]} & {[-0.08+0.02 \eta,-0.04-0.02 \eta]} & {[0.04+0.04 \eta, 0.12-0.04 \eta]} & {[0.22+0.04 \eta, 0.30-0.04 \eta]}
\end{array}\right] \\
\tilde{N}_{2}(\eta)=\left[\begin{array}{cccc}
1 & {[0.07+0.1 \eta, 0.27-0.1 \eta]} & {[-0.35+0.1 \eta,-0.15-0.1 \eta]} & {[0.50+0.04 \eta, 0.58-0.06 \eta]} \\
{[0.44+0.03 \eta, 0.50-0.03 \eta]} & 1 & {[0.65+0.02 \eta, 0.69-0.02 \eta]} & {[-0.34+0.02 \eta,-0.30-0.02 \eta]} \\
{[-0.15+0.04 \eta,-0.07-0.04 \eta]} & {[0.30+0.05 \eta, 0.40-0.05 \eta]} & 1 & {[-0.78+0.04 \eta,-0.70-0.04 \eta]} \\
{[0.50+0.05 \eta, 0.60-0.05 \eta]} & {[0.40+0.03 \eta, 0.46-0.03 \eta]} & {[0.30+0.06 \eta, 0.42-0.06 \eta]} & 1
\end{array}\right]
\end{array}
$$

Further, the parametric fuzzy numbers are changed to its fuzzy-affine form by adopting the procedure given in Section 4. Thus the FNEP with different parameters and the fuzzyaffine coefficients are in the form 


$$
\begin{gathered}
\tilde{N}_{1}\left(\eta, \varepsilon_{i}\right)_{i=1, \cdots, 16}=\left[\begin{array}{cccc}
0.22+(0.02-0.02 \eta) \varepsilon_{1} & 0.02+(0.01-0.01 \eta) \varepsilon_{2} & 0.12+(0.02-0.02 \eta) \varepsilon_{3} & 0.14+(0.01-0.01 \eta) \varepsilon_{4} \\
0.02+(0.01-0.01 \eta) \varepsilon_{5} & 0.14+(0.02-0.02 \eta) \varepsilon_{6} & 0.04+(0.01-0.01 \eta) \varepsilon_{7} & -0.06+(0.02-0.02 \eta) \varepsilon_{8} \\
0.12+(0.02-0.02 \eta) \varepsilon_{9} & 0.04+(0.01-0.01 \eta) \varepsilon_{10} & 0.28+(0.04-0.04 \eta) \varepsilon_{11} & 0.08+(0.04-0.04 \eta) \varepsilon_{12} \\
0.14+(0.01-0.01 \eta) \varepsilon_{13} & -0.06+(0.02-0.02 \eta) \varepsilon_{14} & 0.08+(0.04-0.04 \eta) \varepsilon_{15} & 0.22+(0.02-0.02 \eta) \varepsilon_{16}
\end{array}\right] \\
\tilde{N}_{2}\left(\eta, \varepsilon_{i}\right)_{i=17, \cdots, 28}=\left[\begin{array}{cccc}
1 & 0.17+(0.1-0.1 \eta) \varepsilon_{17} & -0.25+(0.1-0.1 \eta) \varepsilon_{18} & 0.54+(0.04-0.04 \eta) \varepsilon_{19} \\
0.47+(0.03-0.03 \eta) \varepsilon_{20} & 1 & 0.67+(0.02-0.02 \eta) \varepsilon_{21} & -0.32+(0.02-0.02 \eta) \varepsilon_{22} \\
-0.11+(0.04-0.04 \eta) \varepsilon_{23} & 0.35+(0.05-0.05 \eta) \varepsilon_{24} & 1 & -0.74+(0.04-0.04 \eta) \varepsilon_{25} \\
0.55+(0.05-0.05 \eta) \varepsilon_{26} & 0.43+(0.03-0.03 \eta) \varepsilon_{27} & 0.36+(0.06-0.06 \eta) \varepsilon_{28} & 1
\end{array}\right]
\end{gathered}
$$

Now, utilizing the linearization procedure given in Section 5.3, the fuzzy-affine NEP is linearized into fuzzy-affine GEP $\hat{A} \hat{x}^{*}=\hat{\lambda} \hat{B} \hat{x}^{*}$, where

$$
\hat{A}=\left[\begin{array}{c|c}
-\widetilde{N}_{0} & 0 \\
\hline 0 & I
\end{array}\right] \text { and } \hat{B}=\left[\begin{array}{c|c}
\tilde{N}_{1}\left(\eta, \varepsilon_{i}\right)_{i=1, \cdots, 16} & \tilde{N}_{2}\left(\eta, \varepsilon_{i}\right)_{i=17, \cdots, 28} \\
\hline I & 0
\end{array}\right]
$$

Then the fuzzy eigenvalues in the form of TFN are evaluated by adopting the proposed method given in Section 6. Finally, all the fuzzy eigenvalue plots are depicted in Figs. 3(a)-3(h).

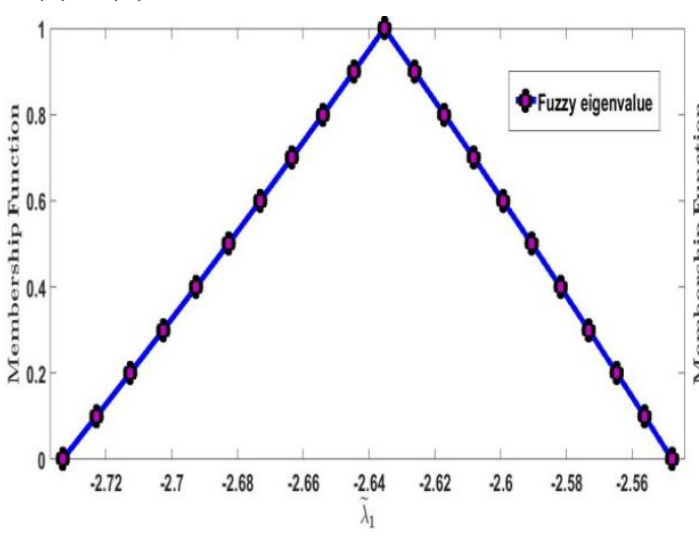

(a)

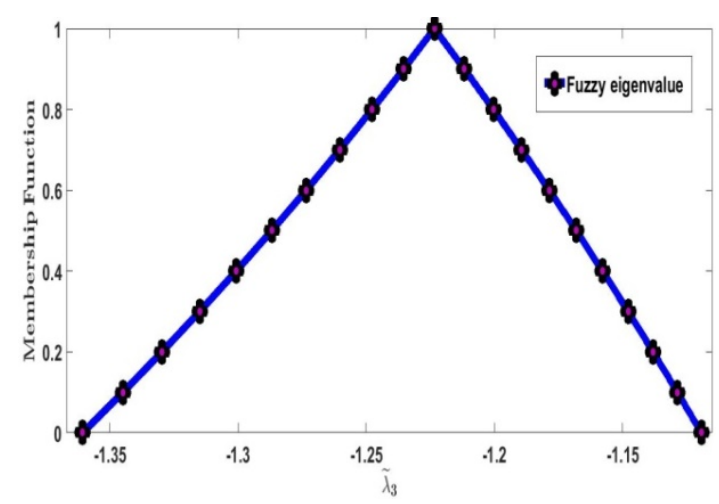

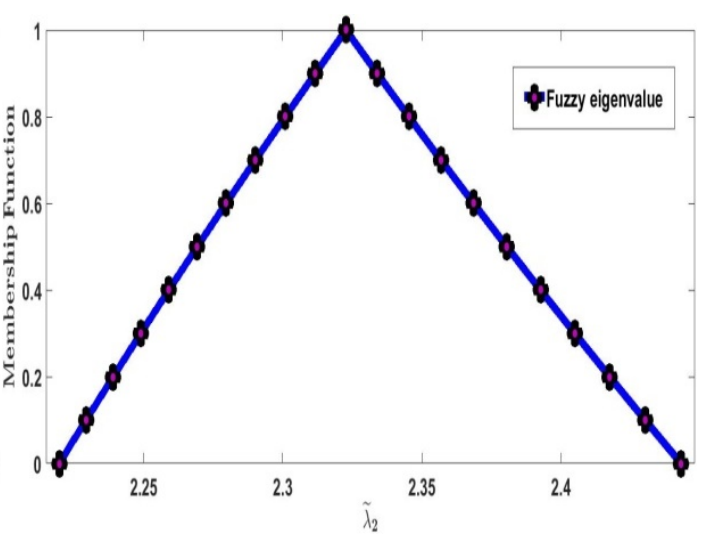

(b)

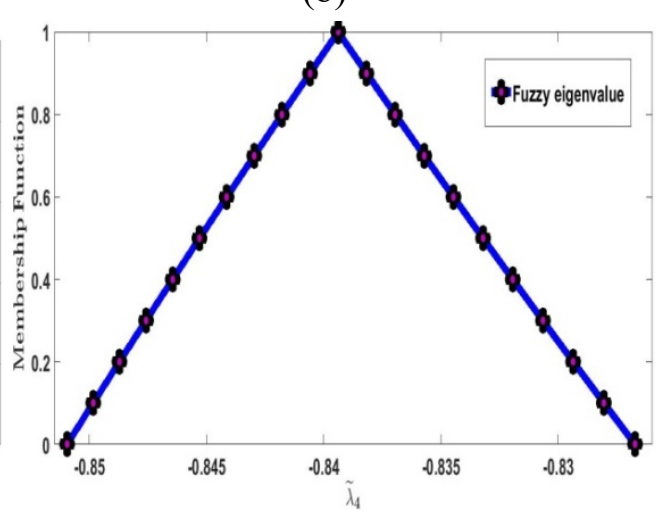


(c)

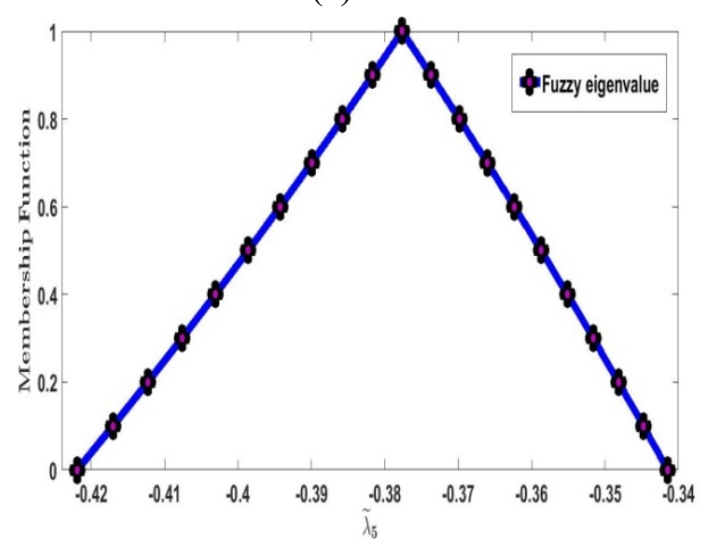

(e)

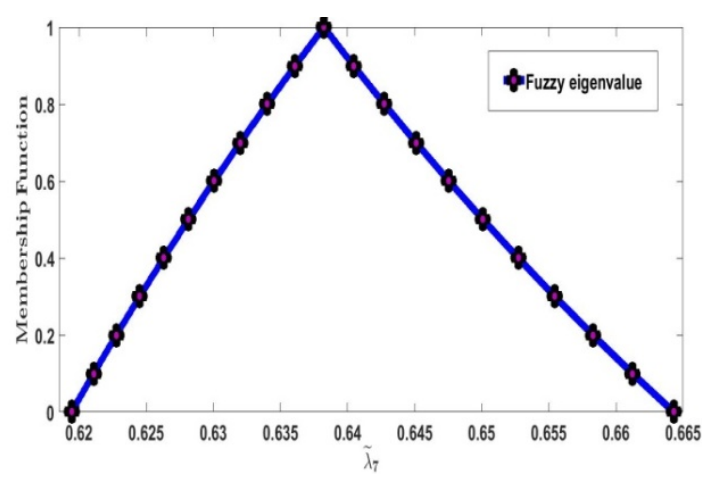

(g) (d)

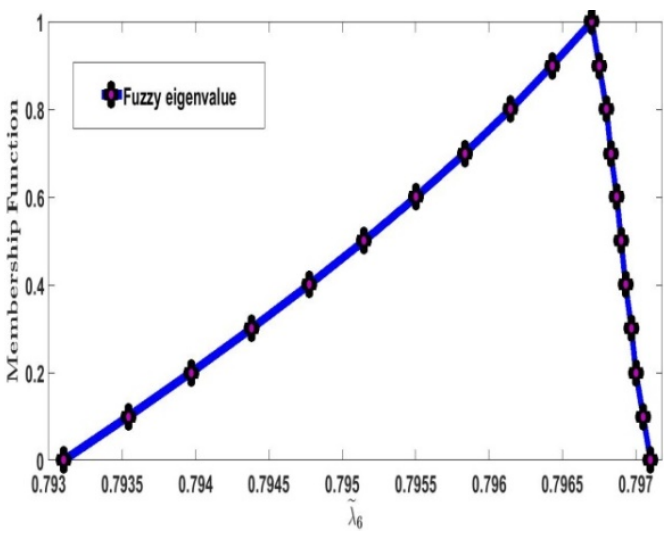

(f)

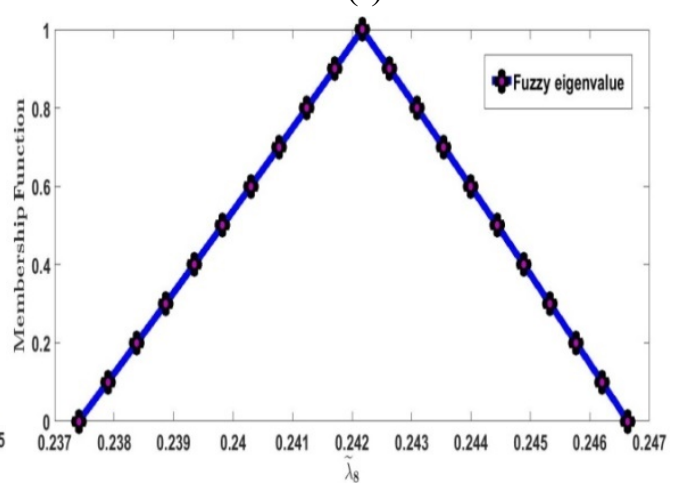

(h)

Figure 3: First-eighth eigenvalue plots for Example 3 (a-h)

Example 4. Let us consider a FNEP $\left(\widetilde{K}+\widetilde{\lambda} \widetilde{C}+\widetilde{\lambda}^{2} \tilde{M}\right) \widetilde{x}=0$ from Eq. (3) of damped spring-mass structural system, where all the coefficient matrices of the problem may be taken as $3 \times 3$ fuzzy matrices having uncertainty in the form of TrFNs. Here the corresponding fuzzy mass, stiffness and damping matrices are given below.

$\widetilde{K}=\left[\begin{array}{ccc}(8.7,8.9,9.1,9.3) & (-3.1,-3.05,-2.95,-2.9) & 0 \\ (-3.1,-3.05,-2.95,-2.9) & (8.7,8.9,9.1,9.3) & (-3.1,-3.05,-2.95,-2.9) \\ 0 & (-3.1,-3.05,-2.95,-2.9) & (8.7,8.9,9.1,9.3)\end{array}\right]$

$\widetilde{C}=\left[\begin{array}{ccc}(11.7,11.9,12.1,12.3) & (-4.1,-4.05,-3.95,-3.9) & 0 \\ (-4.1,-4.05,-3.95,-3.9) & (11.7,11.9,12.1,12.3) & (-4.1,-4.05,-3.95,-3.9) \\ 0 & (-4.1,-4.05,-3.95,-3.9) & (11.7,11.9,12.1,12.3)\end{array}\right]$

and 


$$
\widetilde{M}=\left[\begin{array}{ccc}
(1.8,1.9,2.1,2.2) & 0 & 0 \\
0 & (1.8,1.9,2.1,2.2) & 0 \\
0 & 0 & (1.8,1.9,2.1,2.2)
\end{array}\right]
$$

As worked out in Example 3, here the FNEP is also transformed into fuzzy-affine GEP $\hat{A} \hat{x}^{*}=\hat{\lambda} \hat{B} \hat{x}^{*}$, where

$\hat{A}=\left[\begin{array}{c|c}-K\left(\eta, \varepsilon_{i}\right)_{i=1, \cdots, 7} & 0 \\ \hline 0 & I\end{array}\right]$ and $\hat{A}=\left[\begin{array}{c|c}\widetilde{C}\left(\eta, \varepsilon_{i}\right)_{i=8, \cdots, 14} & \tilde{M}\left(\eta, \varepsilon_{i}\right)_{i=15, \cdots, 17} \\ \hline I & 0\end{array}\right]$.

The above system is solved by using the proposed approach (Section 6) and the fuzzy eigenvalues of the problem are computed. The trapezoidal fuzzy plots of the resulting eigenvalues are illustrated in Figs. 4(a)-4(f).

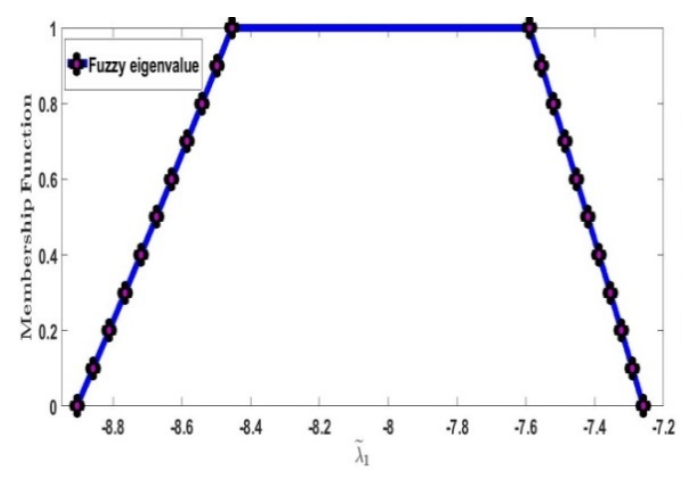

(a)

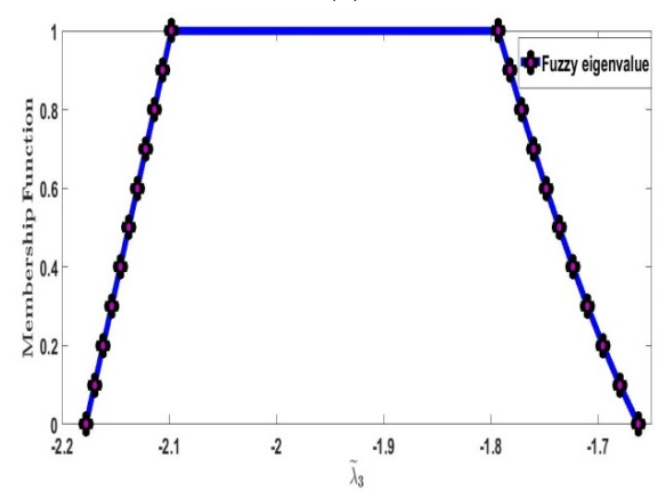

(c)

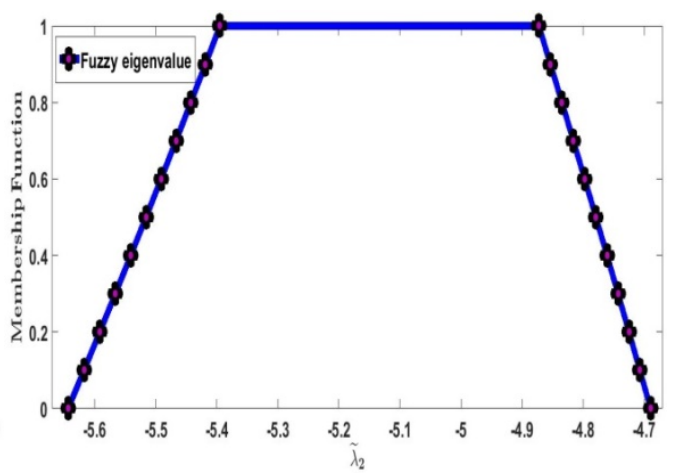

(b)

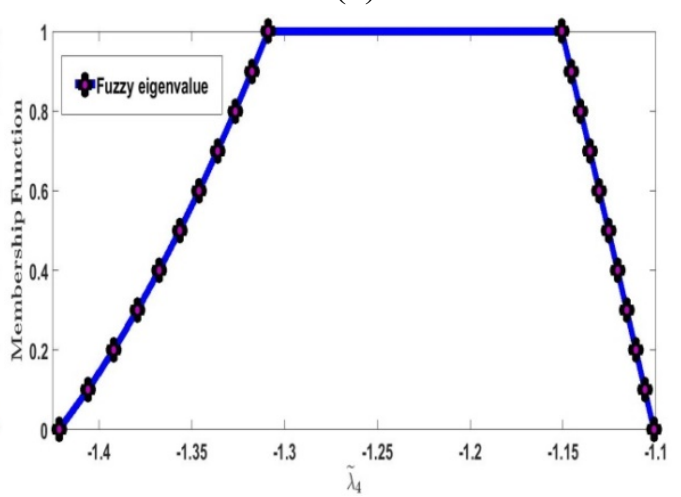

(d) 


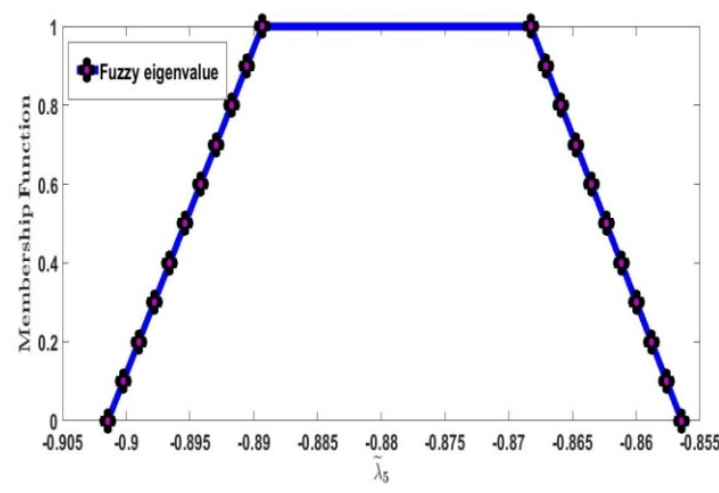

(e)

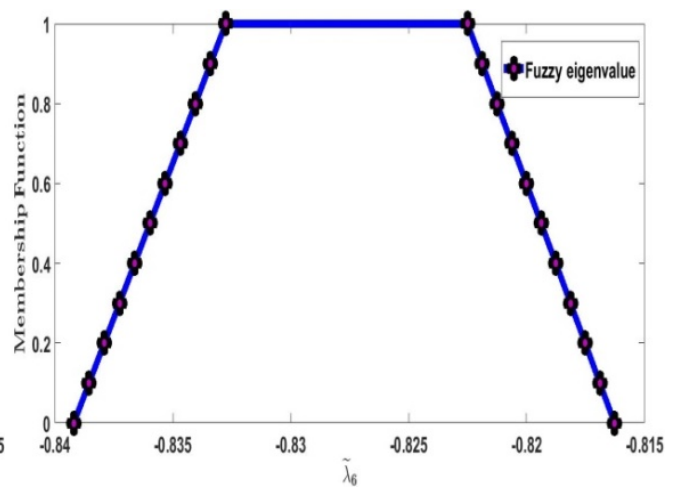

(f)

Figure 4: First to sixth eigenvalue plots for Example 4 (a-f)

Example 5. In Fig. 5, we consider a connected damped spring-mass structural system of ' $n$ ' degrees-of-freedom, where the crisp form is given by Tisseur et al. [Tisseur and Meerbergen (2001)] and interval form is by Chakraverty et al. [Chakraverty and Mahato (2018)]. This system may easily be converted into a NEP of the second degree (that is QEP) $N(\lambda) x=\left(K+C \lambda+M \lambda^{2}\right) x=0$, where $K, C$ and $M$ are the respective stiffness, damping and mass matrices.

According to Fig. 5, $m_{i}$ (ith mass) is connected with $m_{i+1}((i+1) s t$ mass) for $i=1, \cdots, n$ through a spring of stiffness $k_{i}$ and a damper of damping factor $d_{i}$. Further, the ith mass is connected to the ground with spring of stiffness $\kappa_{i}$ and damper of damping factor $\tau_{i}$. Here, the mass matrix is a diagonal matrix such as $M=\operatorname{diag}\left(m_{1}, \cdots, m_{n}\right)$ and both the stiffness and damping matrices are symmetric tridiagonal matrices given by

$$
\begin{aligned}
& K=\Pi \operatorname{diag}\left(k_{1}, \cdots, k_{n-1}, 0\right) \Pi^{T}+\operatorname{diag}\left(\kappa_{1}, \cdots, \kappa_{n}\right), \\
& C=\Pi \operatorname{diag}\left(d_{1}, \cdots, d_{n-1}, 0\right) \Pi^{T}+\operatorname{diag}\left(\tau_{1}, \cdots, \tau_{n}\right),
\end{aligned}
$$

where $\Pi=\left(\delta_{i, j}-\delta_{i, j+1}\right), \delta_{i, j}$ is the 'Kronecker delta'. 


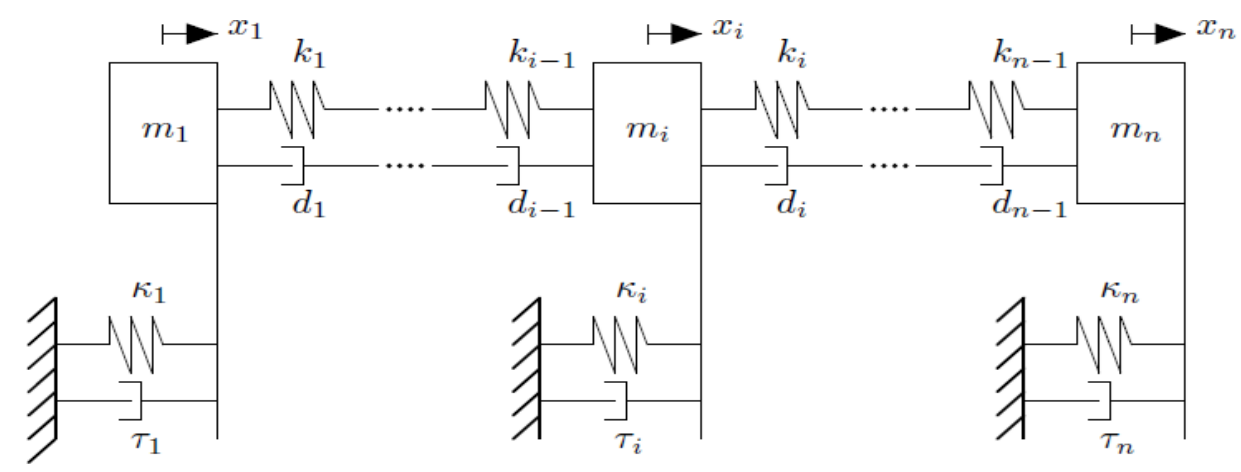

Figure 5: $n$ degrees-of-freedom connected damped spring-mass system

For this particular application problem of structural dynamics, each mass may be weighted as unity (that is $m_{i}=1 \mathrm{~kg}$ for $i=0, \cdots, n$ ). Further, except for the first and last springs, all other springs have a constant stiffness $(\kappa)$ while first and last spring have twice stiffness than others (that is $\kappa_{1}=\kappa_{n}=2 \kappa$ ). Similarly, every damper has also a constant damping factor $(\tau)$ except first and last. The damping factors of the first and last dampers are $\tau_{1}=\tau_{n}=2 \tau$. In this regard, all the involving matrices may be obtained as $K=\kappa \cdot \operatorname{tridiag}(-1,3,-1), C=\tau \cdot \operatorname{tridiag}(-1,3,-1)$ and $M=I_{n \times n}$.

Due to uncertain or vague environments, the dynamic analysis of structural system leads to fuzzy QEP $\tilde{N}(\tilde{\lambda}) \tilde{x}=\left(\widetilde{K}+\tilde{\lambda} \widetilde{C}+\widetilde{\lambda}^{2} \tilde{M}\right) \widetilde{x}=0$, where the coefficients are fuzzy matrices.

Here uncertainties are considered as TFNs. and corresponding stiffness and damping matrices may be written as

$$
\begin{aligned}
\widetilde{K} & =\kappa \cdot \operatorname{tridiag}[(-1.02,-1,-0.98),(2.94,3,3.06),(-1.02,-1,-0.98)] \\
\widetilde{C} & =\tau \cdot \operatorname{tridiag}[(-1.01,-1,-0.99),(2.97,3,3.03),(-1.01,-1,-0.99)] \text { and } \tilde{M}=I_{n \times n} .
\end{aligned}
$$

When the constants for stiffness and damping factor are $\kappa=5$ and $\tau=10$ respectively and the degree-of-freedom be considered as 3 , the following stiffness, damping and mass matrices are formed.

$$
\begin{aligned}
& K^{I}=\left[\begin{array}{ccc}
(14.7,15,15.3) & (-5.1,-5,-4.9) & 0 \\
(-5.1,-5,-4.9) & (14.7,15,15.3) & (-5.1,-5,-4.9) \\
0 & (-5.1,-5,-4.9) & (14.7,15,15.3)
\end{array}\right], \\
& \widetilde{C}=\left[\begin{array}{ccc}
(29.7,30,30.3) & (-10.1,-10,-9.9) & 0 \\
(-10.1,-10,-9.9) & (29.7,30,30.3) & (-10.1,-10,-9.9) \\
0 & (-10.1,-10,-9.9) & (29.7,30,30.3)
\end{array}\right] \text { and } \tilde{M}=I_{3 \times 3} .
\end{aligned}
$$

Adopting the proposed procedure, the above fuzzy QEP $\left(\widetilde{K}+\tilde{\lambda} \widetilde{C}+\tilde{\lambda}^{2} \tilde{M}\right) \widetilde{x}=0$ is converted to fuzzy-affine $\mathrm{QEP}\left(\hat{K}+\hat{\lambda} \hat{C}+\hat{\lambda}^{2} \hat{M}\right) \hat{x}=0$ and finally to fuzzy-affine GEP 
$\hat{A} \hat{x}^{*}=\hat{\lambda} \hat{B} \hat{x}^{*}$ which is solved by using the proposed method. All the fuzzy (TFN) eigenvalue plots are depicted in Figs. 6(a)-6(f).

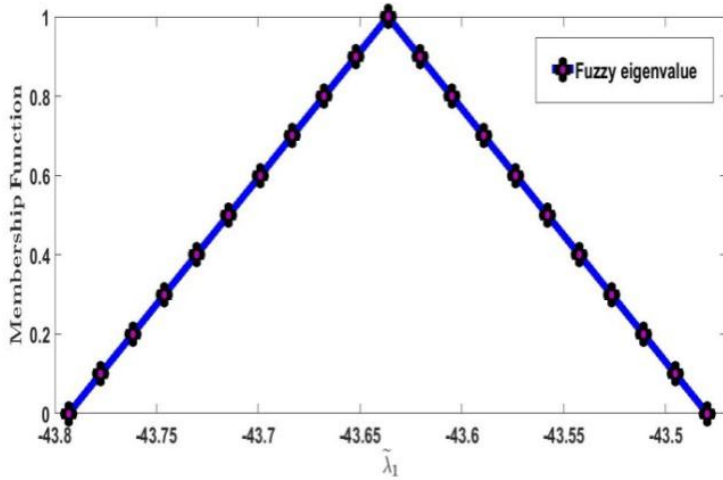

(a)

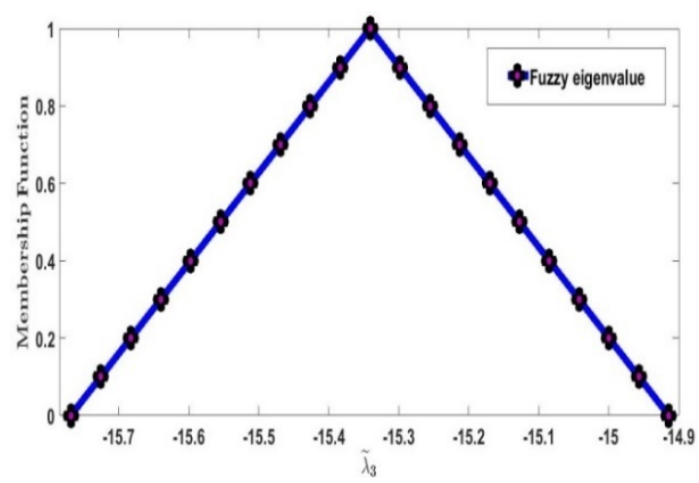

(c)

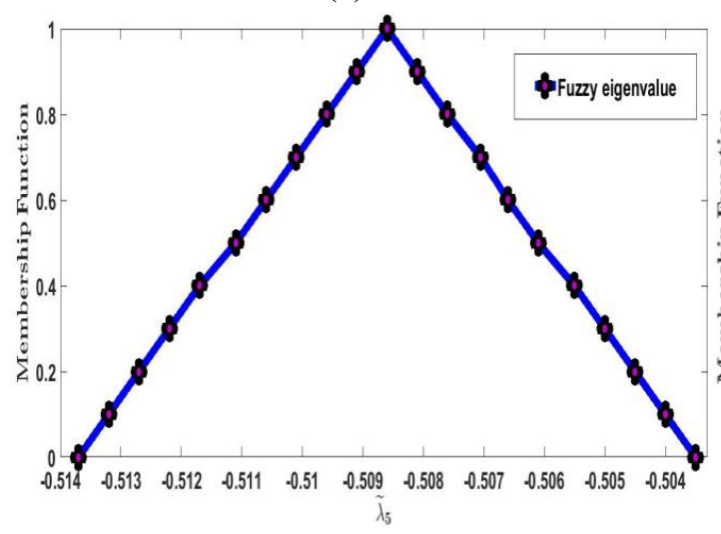

(e)

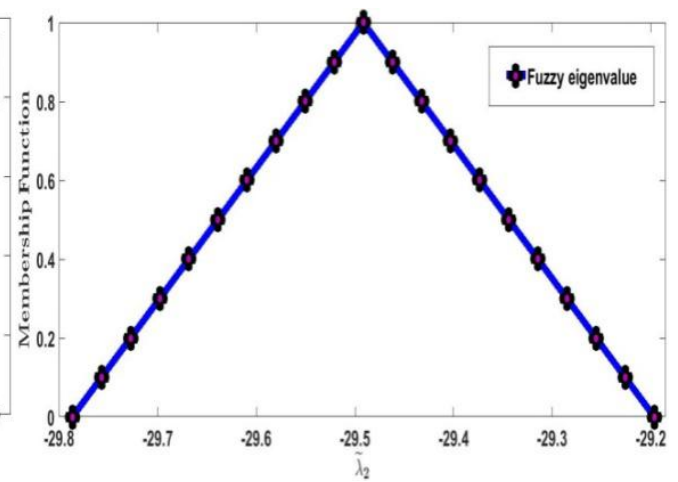

(b)

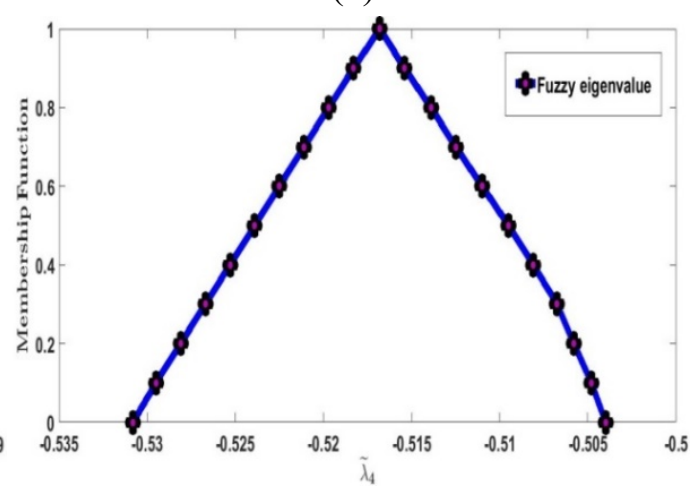

(d)

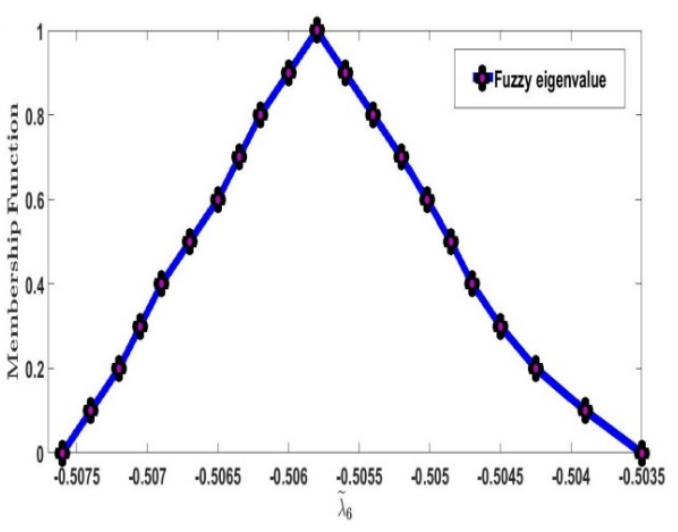

(f)

Figure 6: First to sixth eigenvalue plots for Example 5 (a-f)

Example 6. Further, the above problem has been considered with the TrFNs. Thus the involving matrices are taken in the form as 
$\widetilde{K}=\kappa \cdot \operatorname{tridiag}[(-1.02,-1.01,-0.99,-0.98),(2.94,2.98,3.02,3.06),(-1.02,-1.01,-0.99,-0.98)]$

$\widetilde{C}=\tau \cdot \operatorname{tridiag}[(-1.01,-1.005,-0.995,-0.99),(2.97,2.99,3.01,3.03),(-1.01,-1.005,-0.995,-0.99)]$ and $\tilde{M}=I_{n \times n}$.

Here, the constants of stiffness and damping factor are considered same as Example 5 but the degree-of-freedom is taken as $n=5$. Then the corresponding mass, stiffness and damping matrices may be written as

$$
\begin{aligned}
& \tilde{M}=I_{5 \times 5}, \\
& \tilde{K}=\left[\begin{array}{ccccc}
(14.7,14.9,15.1,15.3) & (-5.1,-5.05,-4.95,-4.9) & 0 & 0 & 0 \\
(-5.1,-5.05,-4.95,-4.9) & (14.7,14.9,15.1,15.3) & (-5.1,-5.05,-4.95,-4.9) & 0 & 0 \\
0 & (-5.1,-5.05,-4.95,-4.9) & (14.7,14.9,15.1,15.3) & (-5.1,-5.05,-4.95,-4.9) & 0 \\
0 & 0 & (-5.1,-5.05,-4.95,-4.9) & (14.7,14.9,15.1,15.3) & (-5.1,-5.05,-4.95,-4.9) \\
0 & 0 & 0 & (-5.1,-5.05,-4.95,-4.9) & (14.7,14.9,15.1,15.3)
\end{array}\right]
\end{aligned}
$$

and

$$
\tilde{C}=\left[\begin{array}{ccccc}
(29.7,29.9,30.1,30.3) & (-10.1,-10.05,-9.95,-9.9) & 0 & 0 & 0 \\
(-10.1,-10.05,-9.95,-9.9) & (29.7,29.9,30.1,30.3) & (-10.1,-10.05,-9.95,-9.9) & 0 & 0 \\
0 & (-10.1,-10.05,-9.95,-9.9) & (29.7,29.9,30.1,30.3) & (-10.1,-10.05,-9.95,-9.9) & 0 \\
0 & 0 & (-10.1,-10.05,-9.95,-9.9) & (29.7,29.9,30.1,30.3) & (-10.1,-10.05,-9.95,-9.9) \\
0 & 0 & 0 & (-10.1,-10.05,-9.95,-9.9) & (29.7,29.9,30.1,30.3)
\end{array}\right]
$$

In the similar fashion as Example 5, the above fuzzy QEP $\left(\widetilde{K}+\tilde{\lambda} \widetilde{C}+\widetilde{\lambda}^{2} \tilde{M}\right) \widetilde{x}=0$ is transformed into fuzzy-affine GEP $\hat{A} \hat{x}^{*}=\hat{\lambda} \hat{B} \hat{x}^{*}$ by utilizing the affine transformation as well as the linearization.

Following the proposed procedure, the trapezoidal fuzzy eigenvalues for the above system are computed. Corresponding fuzzy plots have been included in Figs. 7(a)-7(j).

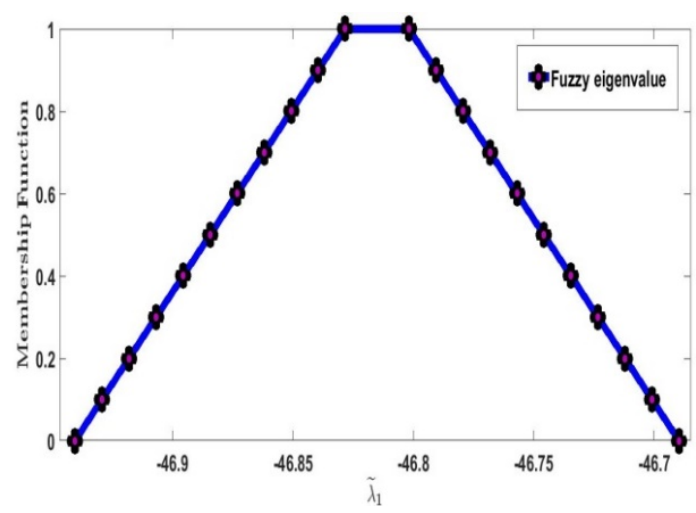

(a)

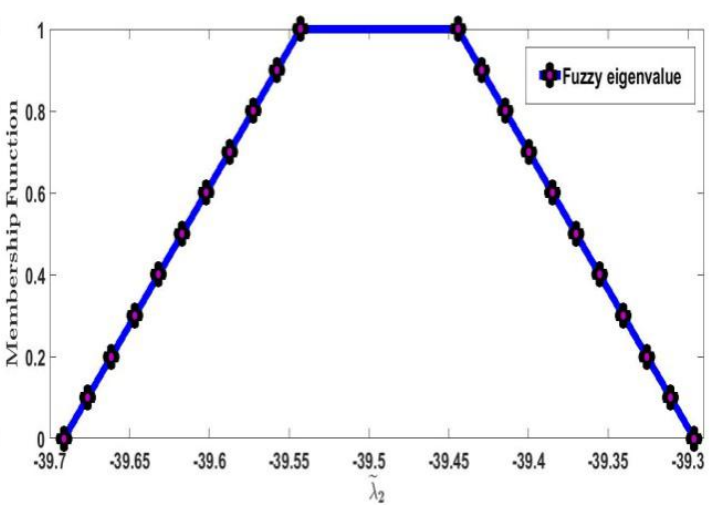

(b) 


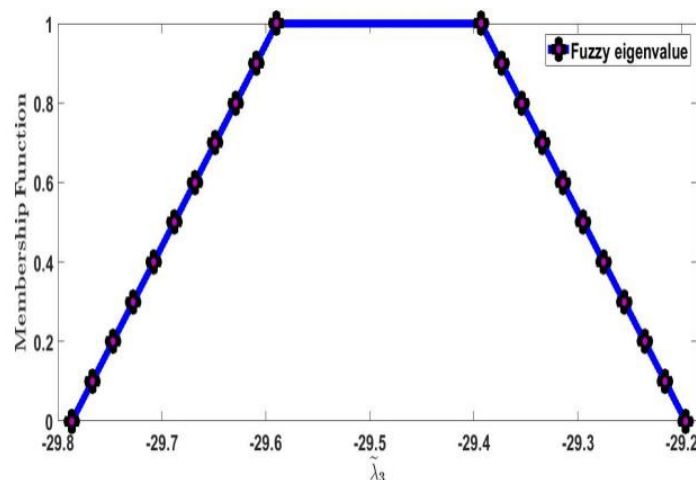

(c)

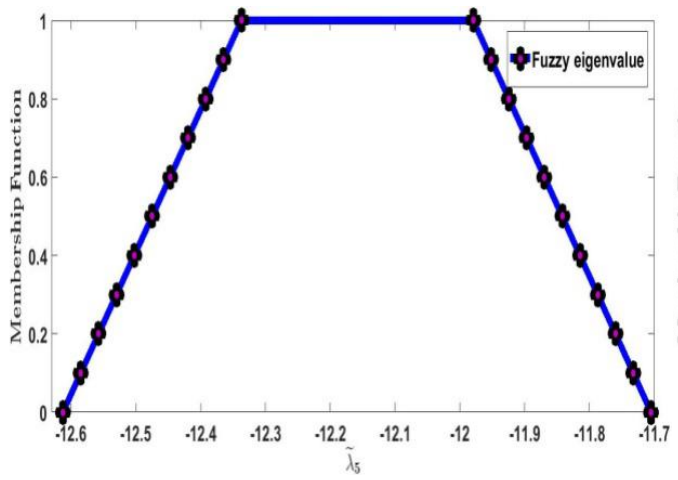

(e)

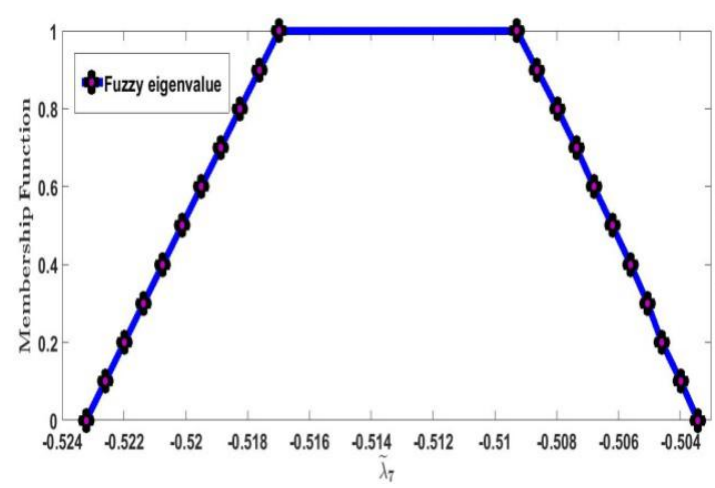

(g)

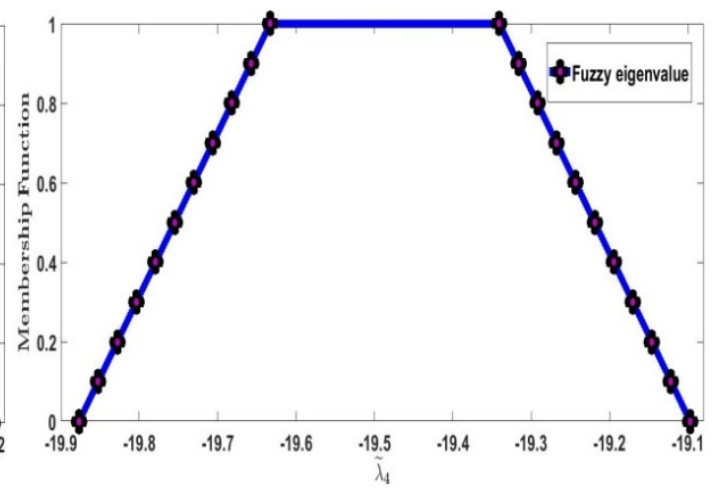

(d)

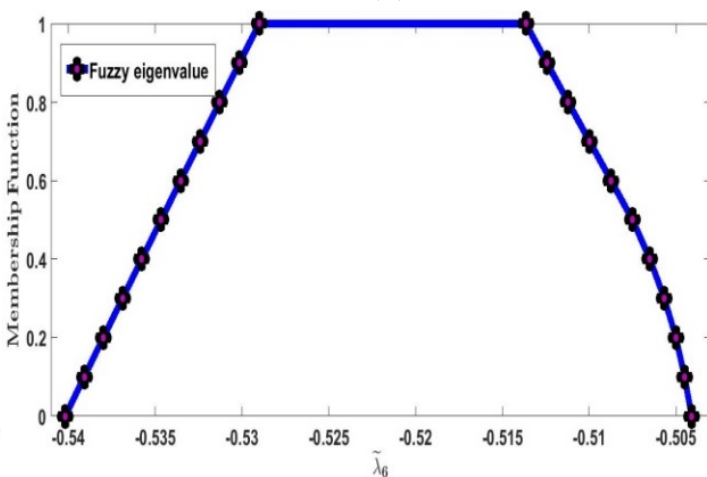

(f)

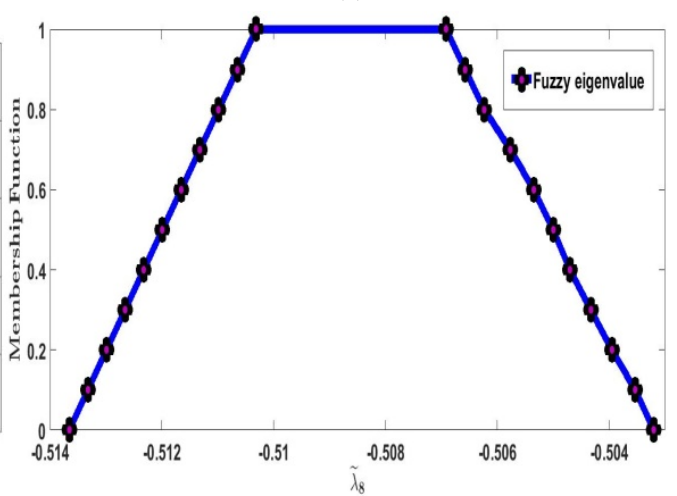

(h) 


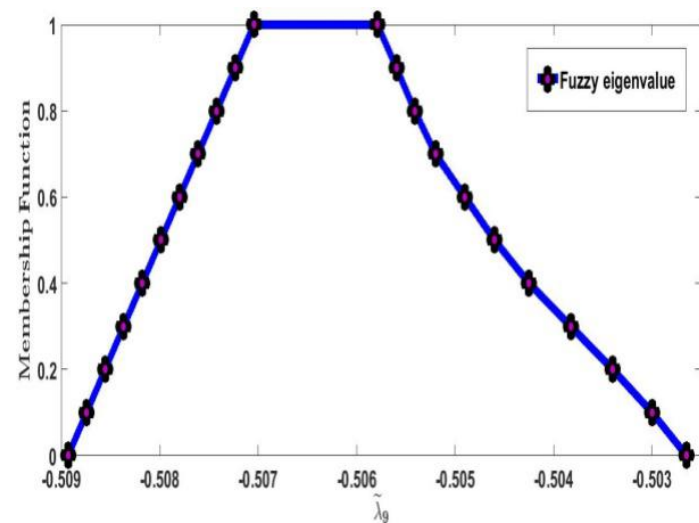

(i)

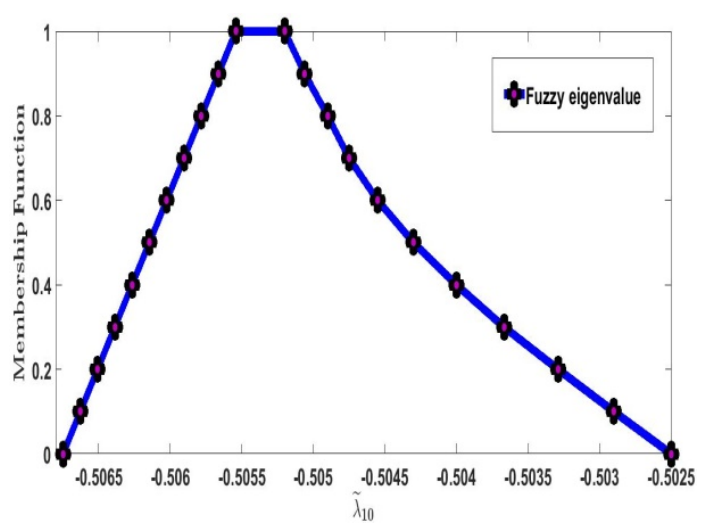

(j)

Figure 7: First to tenth eigenvalue plots for Example $6(\mathrm{a}-\mathrm{j})$

Example 7. Finally, let us consider a fuzzy CEP (interval form given in Chakraverty and Mahato [Chakraverty and Mahato (2018)]) $\tilde{N}(\tilde{\lambda}) \tilde{x}=\left(\widetilde{N}_{0}+\widetilde{N}_{1} \tilde{\lambda}+\widetilde{N}_{2} \widetilde{\lambda}^{2}+\widetilde{N}_{3} \widetilde{\lambda}^{3}\right) \widetilde{x}=0$, where the coefficient matrices are considered as $5 \times 5$ fuzzy matrices (whose the elements are in the form of TFNs) given as follows:

$$
\begin{aligned}
& \widetilde{N}_{0}=\left[\begin{array}{ccccc}
(4.9,5,5.1) & 0 & 0 & 0 & 0 \\
0 & (4.9,5,5.1) & 0 & 0 & 0 \\
0 & 0 & (4.9,5,5.1) & 0 & 0 \\
0 & 0 & 0 & (4.9,5,5.1) & 0 \\
0 & 0 & 0 & 0 & (4.9,5,5.1)
\end{array}\right] \text {, } \\
& \widetilde{N}_{1}=\left[\begin{array}{ccccc}
(8.9,9,9.1) & (-3.1,-3,-2.9) & 0 & 0 & 0 \\
(-3.1,-3,-2.9) & (8.9,9,9.1) & (-3.1,-3,-2.9) & 0 & 0 \\
0 & (-3.1,-3,-2.9) & (8.9,9,9.1) & (-3.1,-3,-2.9) & 0 \\
0 & 0 & (-3.1,-3,-2.9) & (8.9,9,9.1) & (-3.1,-3,-2.9) \\
0 & 0 & 0 & (-3.1,-3,-2.9) & (8.9,9,9.1)
\end{array}\right] \text {, } \\
& \tilde{N}_{2}=\left[\begin{array}{ccccc}
(0.9,1,1.1) & 0 & 0 & (5.9,6,6.1) & 0 \\
0 & (10.4,10.5,10.6) & 0 & 0 & 0 \\
0 & 0 & (0.014,0.015,0.016) & 0 & 0 \\
0 & (250.4,250.5,250.6) & 0 & (-280.1,-280,-279.9) & (33.31,33.32,33.33) \\
0 & 0 & 0 & 0 & (11.9,12,12.1)
\end{array}\right]
\end{aligned}
$$

and 


$\widetilde{N}_{2}=\left[\begin{array}{ccccc}(0.9,1,1.1) & 0 & 0 & (5.9,6,6.1) & 0 \\ 0 & (10.4,10.5,10.6) & 0 & 0 & 0 \\ 0 & 0 & (0.014,0.015,0.016) & 0 & 0 \\ 0 & (250.4,250.5,250.6) & 0 & (-280.1,-280,-279.9) & (33.31,33.32,33.33) \\ 0 & 0 & 0 & 0 & (11.9,12,12.1)\end{array}\right]$

The center eigenvalues of the above fuzzy eigenvalue problem $\left(\widetilde{N}_{0}+\widetilde{N}_{1} \tilde{\lambda}+\widetilde{N}_{2} \widetilde{\lambda}^{2}+\widetilde{N}_{3} \widetilde{\lambda}^{3}\right) \widetilde{x}=0$ have been computed, which yields fifteen eigenvalues out of which seven eigenvalues are real. Accordingly, by using the proposed method, the seven real triangular fuzzy eigenvalues have been evaluated. Corresponding triangular fuzzy plots have been included in Figs. 8(a)-8(g).

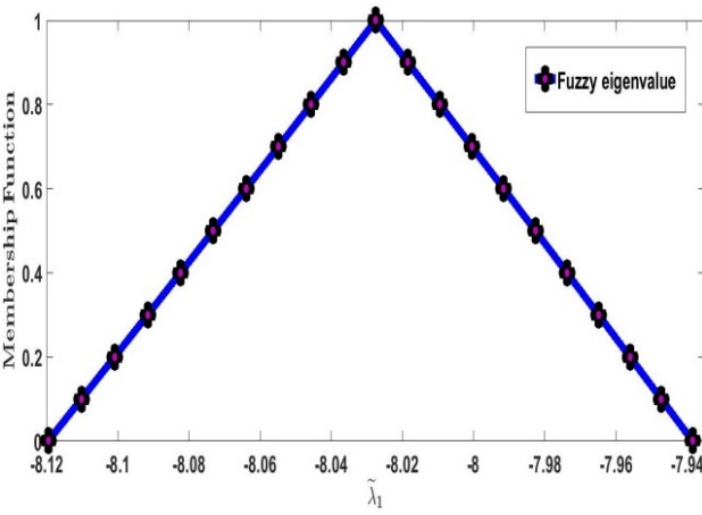

(a)

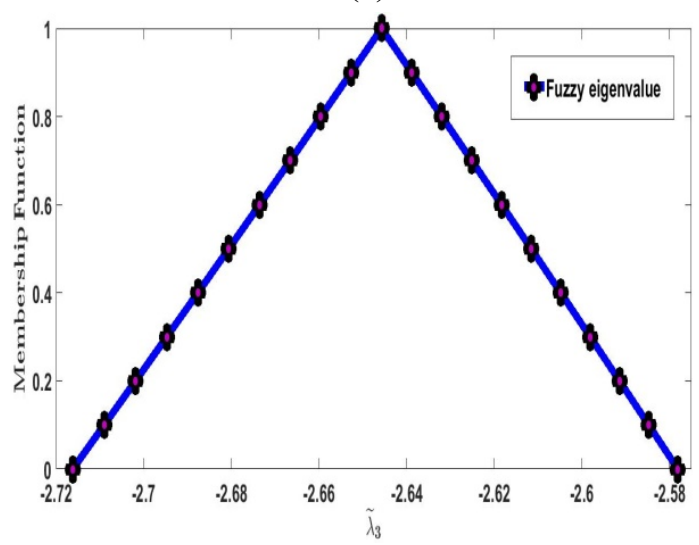

(c)

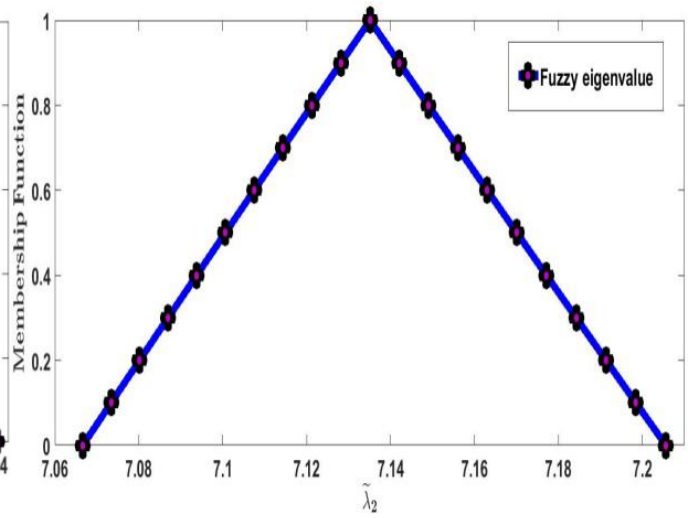

(b)

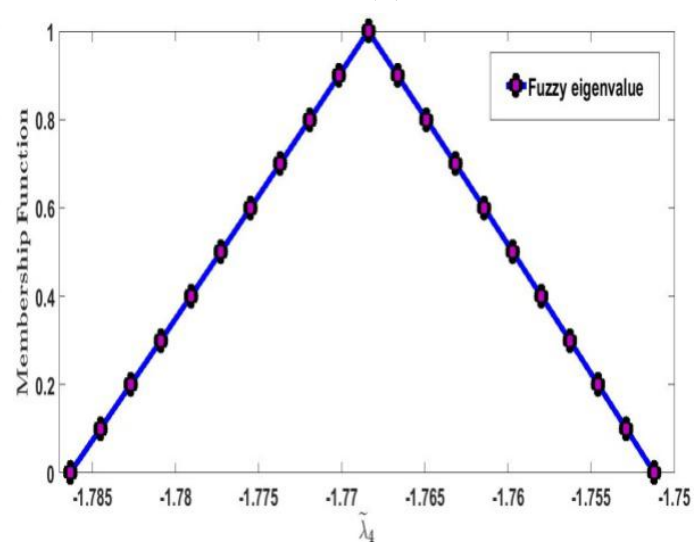

(d) 


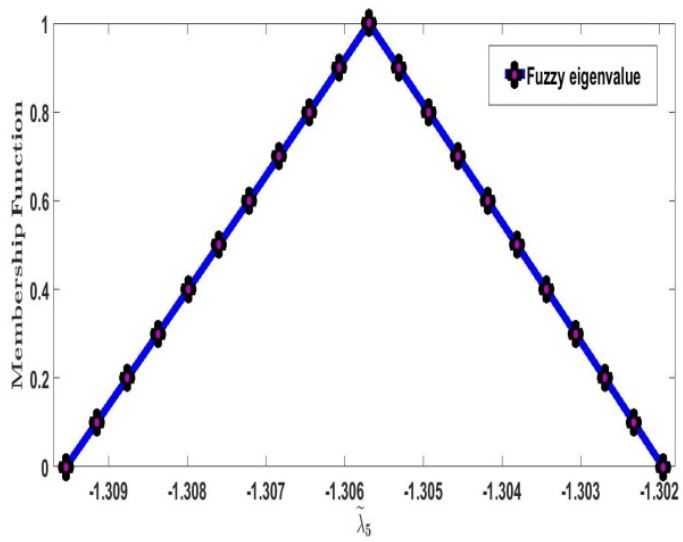

(e)

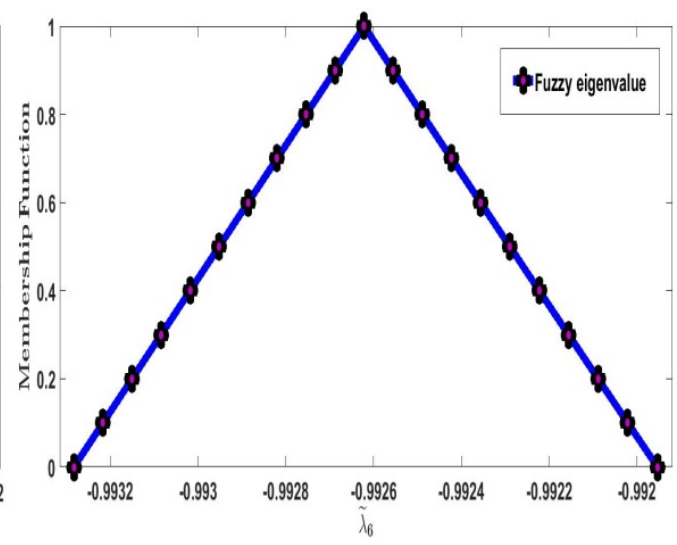

(f)

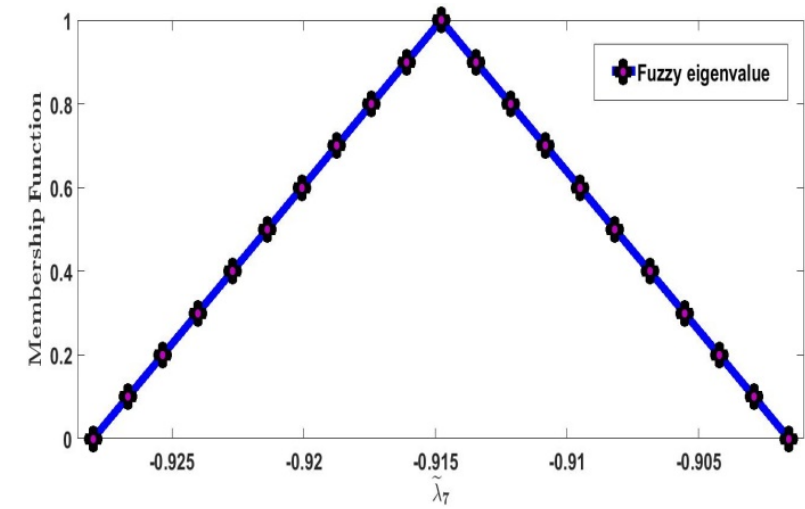

(g)

Figure 8: First to seventh eigenvalue plots for Example 7 (a-g)

\section{Result discussions:}

The center of all the fuzzy eigenvalue solutions (for $\eta=1$ ) of the FNEP given in Example 3 coincides with the crisp eigenvalue solutions of the crisp NEP obtained by Fazeli et al. [Fazeli and Rabiei (2016)] as shown in Tab. 2 and results with tighter enclosures (for $\eta=0$ ). Also for some values of the membership function viz. $\eta=0,0.2$, 0.5, 0.7 and 1, the eigenvalues of Example 3 are listed in Tab. 2. Similarly, we may have that all the central values of the fuzzy eigenvalues in Examples 4 to 6 are equal with the results of NEP for crisp case. Further for Example 6, it may be noticed that the present procedure results in better enclosures for the interval case of NEP as given in Chakraverty et al. [Chakraverty and Mahato (2018)] (for $\eta=0$ ). Moreover, the crisp eigenvalues of the NEP given in Tisseur et al. [Tisseur and Meerbergen (2001)] are same as the center of the trapezoidal fuzzy eigenvalues of Example 6 obtained by substituting $\eta=1$ in the solutions. 
Table 2: Fuzzy eigenvalue bounds of Example 3 for different values of $\eta$

\begin{tabular}{|c|c|c|c|c|c|c|c|c|c|}
\hline Iterations & $\begin{array}{l}\text { Lower } \\
\text { and } \\
\text { upper } \\
\text { bounds }\end{array}$ & $\lambda_{1}$ & $\lambda_{2}$ & $\lambda_{3}$ & $\lambda_{4}$ & $\lambda_{5}$ & $\lambda_{6}$ & $\lambda_{7}$ & $\lambda_{8}$ \\
\hline \multirow{2}{*}{$\eta=0$} & $\underline{\lambda}$ & -2.7332 & 2.2197 & -1.3607 & -0.8510 & -0.4219 & 0.7931 & 0.6194 & 0.2374 \\
\hline & $\bar{\lambda}$ & -2.5478 & 2.4431 & -1.1193 & -0.8267 & -0.3415 & 0.7971 & 0.6643 & 0.2466 \\
\hline \multirow{2}{*}{$\eta=0.2$} & $\underline{\lambda}$ & -2.7126 & 2.2391 & -1.3297 & -0.8487 & -0.4123 & 0.7940 & 0.6228 & 0.2384 \\
\hline & $\bar{\lambda}$ & -2.5647 & 2.4174 & -1.1381 & -0.8294 & -0.3482 & 0.7970 & 0.6583 & 0.2458 \\
\hline \multirow{2}{*}{$\eta=0.5$} & $\underline{\lambda}$ & -2.6828 & 2.2693 & -1.2869 & -0.8453 & -0.3986 & 0.7951 & 0.6281 & 0.2398 \\
\hline & $\bar{\lambda}$ & -2.5905 & 2.3805 & -1.1681 & -0.8332 & -0.3587 & 0.7969 & 0.6501 & 0.2444 \\
\hline \multirow{2}{*}{$\eta=0.7$} & $\lambda$ & -2.6635 & 2.2902 & -1.2604 & -0.8430 & -0.3900 & 0.7958 & 0.6320 & 0.2408 \\
\hline & $\bar{\lambda}$ & -2.6082 & 2.3568 & -1.1893 & -0.8357 & -0.3660 & 0.7968 & 0.6451 & 0.2435 \\
\hline$\eta=1$ & $\underline{\lambda}$ & -2.6353 & 2.3227 & -1.2234 & -0.8394 & -0.3776 & 0.7967 & 0.6382 & 0.2422 \\
\hline$(\underline{\lambda}=\bar{\lambda})$ & $\bar{\lambda}$ & -2.6353 & 2.3227 & -1.2234 & -0.8394 & -0.3776 & 0.7967 & 0.6382 & 0.2422 \\
\hline \multicolumn{2}{|c|}{$\begin{array}{l}\text { Crisp eigenvalues } \\
\text { [Fazeli and Rabiei } \\
\text { (2016)] }\end{array}$} & -2.6353 & 2.3227 & -1.2234 & -0.8394 & -0.3776 & 0.7967 & 0.6382 & 0.2422 \\
\hline
\end{tabular}

Table 3: Comparison of lower and upper fuzzy eigenvalue bounds and its center for Example 7

\begin{tabular}{ccccccc}
\hline \multirow{2}{*}{$\begin{array}{c}\text { Real } \\
\text { Eigenvalues }\end{array}$} & $\begin{array}{c}\text { Lower } \\
(\eta=0)\end{array}$ & $\begin{array}{c}\text { Upper } \\
(\eta=0)\end{array}$ & $\begin{array}{c}\text { Center } \\
(\eta=1)\end{array}$ & Lower & Upper & Center \\
\hline$\lambda_{1}$ & -8.1195 & -7.9384 & -8.0275 & -8.1495 & -7.9092 & -8.0275 \\
$\lambda_{2}$ & 7.0667 & 7.2056 & 7.1351 & 7.0638 & 7.2084 & 7.1351 \\
$\lambda_{3}$ & -2.7162 & -2.5781 & -2.6457 & -2.7669 & -2.5299 & -2.6457 \\
$\lambda_{4}$ & -1.7863 & -1.7512 & -1.7684 & -1.8320 & -1.7073 & -1.7684 \\
$\lambda_{5}$ & -1.3095 & -1.3020 & -1.3057 & -1.3399 & -1.2738 & -1.3057 \\
$\lambda_{6}$ & -0.9280 & -0.9016 & -0.9148 & -0.9438 & -0.8901 & -0.9148 \\
$\lambda_{7}$ & -0.9920 & -0.9933 & -0.9926 & -0.9950 & -0.9885 & -0.9926 \\
\hline
\end{tabular}


Moreover, the results of fuzzy CEP (for $\eta=0$ ) of Example 7 are compared with the interval eigenvalue bounds of the interval CEP of Chakraverty et al. [Chakraverty and Mahato (2018)] in Tab. 3 and are found to be in good agreement. It may be noted from Tab. 3 that the present approach gives comparatively tighter bounds than Chakraverty et al. [Chakraverty and Mahato (2018)] and the center eigenvalues for both the cases are coincide with each other [Chakraverty and Mahato (2018)].

\section{Conclusion}

In this article, an efficient fuzzy-affine approach (FAA) has been proposed to solve fully FNEPs of damped spring-mass structural system. FAA is developed to overcome the overestimation problems. FAA is found to give better enclosures than basic FA. Further, by utilizing fuzzy-affine form, the FNEP is transformed into a form of NEP having different parameters in terms of noise symbols and membership functions which may easily be linearized to GEP. Fuzzy eigenvalues are computed by varying the parameters $\varepsilon_{i}$ from -1 to 1 and $\eta$ from 0 to 1 . Several examples of damped spring-mass structural problems with other quadratic and cubic eigenvalue problems are included to show the powerfulness and efficacy of the proposed procedure.

\section{References}

Adusumilli, B. S.; Kumar, B. K. (2018): Modified affine arithmetic based continuation power flow analysis for voltage stability assessment under uncertainty. IET Generation, Transmission \& Distribution, vol. 12, no. 18, pp. 4225-4232.

Akhmerov, R. R. (2005): Interval-affine Gaussian algorithm for constrained systems. Reliable Computing, vol. 11, no. 5, pp. 323-341.

Apel, T.; Mehrmann, V.; Watkins, D. (2002): Structured eigenvalue methods for the computation of corner singularities in 3D anisotropic elastic structures. Computer Methods in Applied Mechanics and Engineering, vol. 191, no. 39-40, pp. 4459-4473.

Bai, Z.; Su, Y. (2005): SOAR: a second-order Arnoldi method for the solution of the quadratic eigenvalue problem. SIAM Journal on Matrix Analysis and Applications, vol. 26, no. 3, pp. 640-659.

Bender, C. M.; Komijani, J.; Wang, Q. H. (2019): Nonlinear eigenvalue problems for generalized Painlevé equations. Journal of Physics A: Mathematical and Theoretical, vol. 52, no. 31 .

Chakraverty, S.; Mahato, N. R. (2018): Nonlinear interval eigenvalue problems for damped spring-mass system. Engineering Computations, vol. 35, no. 6, pp. 2272-2286.

Chakraverty, S.; Perera, S. (2018): Recent Advances in Applications of Computational and Fuzzy Mathematics. Springer Nature Singapore.

Chakraverty, S.; Tapaswini, S.; Behera, D. (2016): Fuzzy Differential Equations and Applications for Engineers and Scientists. CRC Press.

Comba, J. L. D.; Stol, J. (1993): A ne arithmetic and its applications to computer graphics. Proceedings of VI SIBGRAPI (Brazilian Symposium on Computer Graphics and Image Processing), pp. 9-18. 
De Figueiredo, L. H.; Stolfi, J. (2004): Affine arithmetic: concepts and applications. Numerical Algorithms, vol. 37, no. 1-4, pp. 147-158.

Dubois, D. J. (1980): Fuzzy Sets and Systems: Theory and Applications, vol. 144. Academic Press.

Fazeli, S. A.; Rabiei, F. (2016): Solving nonlinear eigenvalue problems using an improved newton method. International Journal of Advanced Computer Science and Applications, vol. 7, no. 9, pp. 438-441.

Gao, W.; Yang, C.; Meza, J. C. (2009): Solving a Class of Nonlinear Eigenvalue Problems by Newton's Method (No. LBNL-2187E). Lawrence Berkeley National Lab. (LBNL), Berkeley, CA (United States).

Gohberg, I.; Lancaster, P.; Rodman, L. (1982): Matrix Polynomials. Acad. Press, New York.

Hanss, M. (2005): Applied Fuzzy Arithmetic: An Introduction with Engineering Applications/Hanss M, vol. 1, pp. 100-116. Springer.

Hladik, M.; Daney, D.; Tsigaridas, E. (2011): A filtering method for the interval eigenvalue problem. Applied Mathematics and Computation, vol. 217, no. 12, pp. 5236-5242.

Kaufmann, A.; Gupta, M. M. (1988): Fuzzy Mathematical Models in Engineering and Management Science. Elsevier Science Inc.

Kressner, D. (2009): A block Newton method for nonlinear eigenvalue problems. Numerische Mathematik, vol. 114, no. 2, pp. 355-372.

Kurseeva, V. Y.; Tikhov, S. V.; Valovik, D. V. (2019): Nonlinear multiparameter eigenvalue problems: linearised and nonlinearised solutions. Journal of Differential Equations, vol. 267, no. 4, pp. 2357-2384.

Lawrence, P. W.; Van Barel, M.; Van Dooren, P. (2016): Backward error analysis of polynomial eigenvalue problems solved by linearization. SIAM Journal on Matrix Analysis and Applications, vol. 37, no. 1, pp. 123-144.

Leng, H. (2014): Real eigenvalue bounds of standard and generalized real interval eigenvalue problems. Applied Mathematics and Computation, vol. 232, pp. 164-171.

Mahato, N. R.; Chakraverty, S. (2016a): Filtering algorithm for real eigenvalue bounds of interval and fuzzy generalized eigenvalue problems. ASME Journal of Risk and Uncertainty in Engineering Systems, Part B: Mechanical Engineering, vol. 2, no. 4, 044502.

Mahato, N. R.; Chakraverty, S. (2016b): Filtering algorithm for eigenvalue bounds of fuzzy symmetric matrices. Engineering Computations, vol. 33, no. 3, pp. 855-875.

Mehrmann, V.; Watkins, D. (2002): Polynomial eigenvalue problems with Hamiltonian structure. Electronic Transactions on Numerical Analysis, vol. 13, pp. 106-118.

Miyajima, S.; Kashiwagi, M. (2004): A dividing method utilizing the best multiplication in affine arithmetic. IEICE Electronics Express, vol. 1, no. 7, pp. 176-181.

Przemieniecki, J. S. (1985): Theory of Matrix Structural Analysis. Courier Corporation. 
Rajakumar, C. (1993): Lanczos algorithm for the quadratic eigenvalue problem in engineering applications. Computer Methods in Applied Mechanics and Engineering, vol. 105, no. 1, pp. 1-22.

Rex, G.; Rohn, J. (1998): Sufficient conditions for regularity and singularity of interval matrices. SIAM Journal on Matrix Analysis and Applications, vol. 20, no. 2, pp. 437-445.

Romero-Quete, D.; Cañizares, C. A. (2018): An affine arithmetic-based energy management system for isolated microgrids. IEEE Transactions on Smart Grid, vol. 10, no. 3, pp. 2989-2998.

Rump, S. M.; Kashiwagi, M. (2015): Implementation and improvements of affine arithmetic. Nonlinear Theory and Its Applications, IEICE, vol. 6, no. 3, pp. 341-359.

Saad, Y.; El-Guide, M.; Miedlar, A. (2019): A rational approximation method for the nonlinear eigenvalue problem. arXiv:1901.01188.

Sadangi, S. (2013): Interval Nonlinear Eigenvalue Problems (Ph.D. Thesis). National Institute of Technology Rourkela, India.

Skalna, I. (2009): Direct method for solving parametric interval linear systems with nonaffine dependencies. International Conference on Parallel Processing and Applied Mathematics, pp. 485-494.

Skalna, I.; Hladík, M. (2017): A new algorithm for Chebyshev minimum-error multiplication of reduced affine forms. Numerical Algorithms, vol. 76, no. 4, pp. 1131-1152.

Solov'ev, S. I.; Solov'ev, P. S. (2018): Finite element approximation of the minimal Eigenvalue of a nonlinear Eigenvalue problem. Lobachevskii Journal of Mathematics, vol. 39, no. 7, pp. 949-956.

Stolfi, J.; De Figueiredo, L. H. (2003): An introduction to affine arithmetic. Trends in Applied and Computational Mathematics, vol. 4, no. 3, pp. 297-312.

Su, Y.; Bai, Z. (2011): Solving rational eigenvalue problems via linearization. SIAM Journal on Matrix Analysis and Applications, vol. 32, no. 1, pp. 201-216.

Tisseur, F. (2000): Backward error and condition of polynomial eigenvalue problems. Linear Algebra and its Applications, vol. 309, no. 1-3, pp. 339-361.

Tisseur, F.; Meerbergen, K. (2001): The quadratic eigenvalue problem. SIAM Review, vol. 43, no. 2, pp. 235-286.

Wang, S.; Wang, K.; Teng, F.; Strbac, G.; Wu, L. (2018): An affine arithmetic-based multi-objective optimization method for energy storage systems operating in active distribution networks with uncertainties. Applied Energy, vol. 223, pp. 215-228.

Wetherhold, R.; Padliya, P. S. (2014): Design aspects of nonlinear vibration analysis of rectangular orthotropic membranes. Journal of Vibration and Acoustics, vol. 136, no. 3, 034506.

Xu, C.; Gu, W.; Gao, F.; Song, X.; Meng, X. et al. (2016): Improved affine arithmetic based optimisation model for interval power flow analysis. IET Generation, Transmission \& Distribution, vol. 10, no. 15, pp. 3910-3918.

Zadeh, L. A. (1965): Fuzzy sets. Information and Control, vol. 8, no. 3, pp. 338-353. 
Zadeh, L. A.; Fu, K. S.; Tanaka, K. (2014): Fuzzy sets and their applications to cognitive and decision processes. Proceedings of the US-Japan Seminar on Fuzzy Sets and Their Applications.

Zimmermann, H. J. (2011): Fuzzy Set Theory-and Its Applications. Springer Science \& Business Media. 